\title{
Physical model of near-Earth asteroid (1917) Cuyo from ground-based optical and thermal-IR observations ${ }^{\star, \star \star}$
}

\author{
A. Rożek ${ }^{1}$, S. C. Lowry ${ }^{1}$, B. Rozitis ${ }^{2}$, S. F. Green ${ }^{2}$, C. Snodgrass ${ }^{3,2}$, P. R. Weissman 4 , A. Fitzsimmons ${ }^{5}$, M. D. Hicks ${ }^{6}$,
} K. J. Lawrence ${ }^{6}$, S. R. Duddy ${ }^{1}$, S. D. Wolters ${ }^{2}$, G. Roberts-Borsani ${ }^{1,7}$, R. Behrend ${ }^{8}$, and F. Manzini ${ }^{9}$

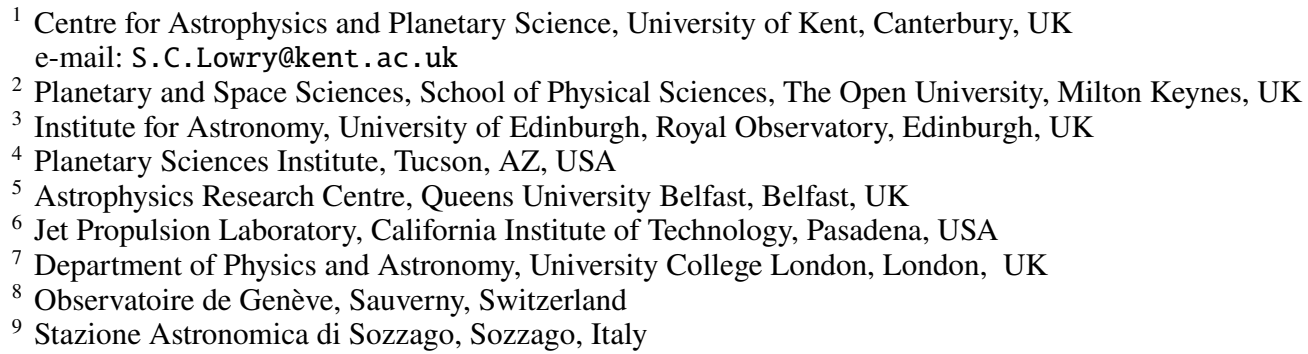

Received 30 August 2018 / Accepted 3 May 2019

\begin{abstract}
Context. The near-Earth asteroid (1917) Cuyo was subject to radar and light curve observations during a close approach in 1989, and observed up until 2008. It was selected as one of our ESO Large Programme targets, aimed at observational detections of the Yarkovsky-O'Keefe-Radzievskii-Paddack (YORP) effect through long-term light curve monitoring and physical modelling of nearEarth asteroids.

Aims. We aim to constrain the physical properties of Cuyo: shape, spin-state, and spectroscopic and thermo-physical properties of the surface.

Methods. We acquired photometric light curves of Cuyo spanning the period between 2010 and 2013, which we combined with published light curves from 1989 to 2008 . Our thermal-infrared observations were obtained in 2011. Rotationally resolved optical spectroscopy data were acquired in 2011 and combined with all available published spectra to investigate any surface material variegation. Results. We developed a convex light curve-inversion shape of Cuyo that suggests the presence of an equatorial ridge, typical for an evolved system close to shedding mass due to fast rotation. We determine limits of YORP strength through light curvebased spin-state modelling, including both negative and positive acceleration values, between $-0.7 \times 10^{-8}$ and $1.7 \times 10^{-8} \mathrm{rad} \mathrm{day}^{-2}$. Thermo-physical modelling with the ATPM provides constraints on the geometric albedo, $p_{\mathrm{V}}=0.24 \pm 0.07$, the effective diameter, $D_{\text {eff }}=3.15 \pm 0.08 \mathrm{~km}$, the thermal inertia, $\Gamma=44 \pm 9 \mathrm{~J} \mathrm{~m}^{-2} \mathrm{~s}^{-1 / 2} \mathrm{~K}^{-1}$, and a roughness fraction of $0.52 \pm 0.26$. This enabled a YORP strength prediction of $v=(-6.39 \pm 0.96) \times 10^{-10} \mathrm{rad} \mathrm{day}^{-2}$. We also see evidence of surface compositional variation.

Conclusions. The low value of YORP predicted by means of thermo-physical analysis, consistent with the results of the light curve study, might be due to the self-limiting properties of rotational YORP, possibly involving movement of sub-surface and surface material. This may also be consistent with the surface compositional variation that we see. The physical model of Cuyo can be used to investigate cohesive forces as a way to explain why some targets survive rotation rates faster than the fission limit.
\end{abstract}

Key words. minor planets, asteroids: individual: Cuyo - methods: observational - radiation mechanisms: thermal techniques: photometric - techniques: spectroscopic - methods: data analysis

\section{Introduction}

The Yarkovsky-O'Keefe-Radzievskii-Paddack (YORP) effect is a small torque produced by reflection and thermal re-radiation of absorbed sunlight by an asteroid's surface (Rubincam 2000). It can change the orientation of the spin axis relative to the orbital plane, as well as affect the rotation period. It is currently accepted to be the main driver of spin-state evolution for nearEarth asteroids (NEAs). The YORP-induced spin-up of rotation can affect the physical shape of rubble pile asteroids, including

\footnotetext{
* Tables A.1 and A.2 are only available at the CDS via anonymous ftp to cdsarc.u-strasbg. fr (130.79.128.5) or via http://cdsarc. u-strasbg.fr/viz-bin/qcat?]/A+A/627/A172

$\star \star$ Based in part on observations collected at the European Southern Observatory, Chile, under programmes 185.C-1033 and 185.C-1034.
}

the peculiar "spinning-top" or "YORPoid" shape of many asteroids, first seen on asteroid (66391) $1999 \mathrm{KW}_{4}$ (Ostro et al. 2006; Scheeres et al. 2006). Eventually, YORP-induced spin-up can increase the rotation rate to the spin fission limit, leading to break up and the creation of a binary asteroid system, or an unbound pair (Walsh et al. 2008, 2012). YORP is coupled with the Yarkovsky effect to influence the orbital evolution of small asteroids, aiding the delivery of small asteroids to the near-Earth region (Rubincam 2000; Bottke et al. 2002; Chesley et al. 2003).

The first direct detection of a YORP-induced spin-rate acceleration was reported for NEA (54509) YORP (initial designation $\left.2000 \mathrm{PH}_{5}\right)$ using combined analysis of light curve and radar data (Lowry et al. 2007; Taylor et al. 2007). The YORPinduced spin-up has been confirmed for only five other objects: (1862) Apollo (Kaasalainen et al. 2007), (1620) Geographos 
(Ďurech et al. 2008), (3103) Eger (Ďurech et al. 2012), (25143) Itokawa (Lowry et al. 2014), and most recently on (161989) Cacus (Ďrech et al. 2018). The notable feature of all reported values to date is that they are in spin-up mode. Recent theoretical advancements in this field are providing a good description of how YORP operates (e.g. Rozitis \& Green 2013); these advancements predict spin-up and spin-down modes. Other recent studies invoke a "Tangential YORP" torque to account for the absence of spin-down modes. Real surfaces are not flat, but are strewn with large boulders. Thermal conduction through these features may lead to thermal emission on the other side of them, producing a recoil force in a direction tangential to the surface (Golubov et al. 2014). Considering the need for more direct detections to improve agreement between the models and observations, we are conducting a long-term observing campaign, primarily through a European Southern Observatory Large Programme (ESO LP). The ESO LP observing campaign was carried out mainly at optical wavelengths with the ESO Faint Object Spectrograph and Camera v.2 (EFOSC2) instrument at the $3.6 \mathrm{~m}$ New Technology Telescope (NTT) in ESO's La Silla observatory in Chile in the 2010 to 2014 period. The photometric monitoring was conducted, and continues, at other ground-based facilities in concert with the ESO LP observations, allowing us to broaden the light curve coverage for our sample.

A selection of 42 NEAs have been observed since April 2010. The objects were selected for the programme based on properties that make them likely candidates for new YORP detections: they have short rotation periods, sizes that are sufficiently small, and orbits close to the Sun. Their short rotation periods also make them convenient photometry targets; observations covering a full rotation can be obtained with just a few hours of continuous monitoring or through folding light curve segments obtained during just a few consecutive days of observations The orbital geometries of our sample allow for regular revisiting. This long-term light curve monitoring with optical facilities enables detections of YORP-induced rotation period changes.

The observations from NTT were supplemented with thermal-infrared spectra from the VLT Imager and Spectrometer for mid Infrared (VISIR) instrument at the $8.2 \mathrm{~m}$ Very Large Telescope (VLT) in ESO's Paranal Observatory in Chile. The infrared observations are complementary to the light curve studies, and allow for better constraints on predictions of YORP for any target by deriving thermo-physical parameters such as surface roughness and thermal inertia. When possible, the optical light curve data set is complemented by radar observations from the Arecibo and Goldstone observatories.

In this study we aim to produce a physical model of the asteroid (1917) Cuyo, hereafter referred to as Cuyo, using data from the ESO LP and supporting campaign, and search for possible signatures of a YORP-induced spin-state change. Cuyo, initially designated 1968 AA, belongs to the Amor group of NEAs, with a semi-major axis of 2.15 AU and eccentricity 0.51. Cuyo could be a potential target for future spacecraft missions (Shoemaker \& Helin 1978), given the low velocity impulse required to put a spacecraft on a rendezvous orbit with the asteroid, $\Delta V \approx$ $8.6 \mathrm{~km} \mathrm{~s}^{-1}$. The object has an extensive literature light curve data set, spanning the period from 1989 to 2008. The near-Earth orbit, estimated size, and long time-base of archive observations made it a likely target for a YORP-induced rotation period change detection.

Cuyo was first observed extensively during its approach in 1989 when it passed Earth within 0.1 AU. It was detected with the planetary radar at the Arecibo Observatory in September 1989 and with Goldstone radar in October 1989 (Ostro \& Wisniewski 1992). The radar observations were linked with optical photometric light curves taken with the $2.3 \mathrm{~m}$ Steward Observatory telescope at Kitt Peak, which allowed an estimate of the synodic rotation period of $P=2.693 \mathrm{~h}$ (Wisniewski et al. 1997). The early convex-profile light curve inversion modelling revealed a "quasi-triangular" pole-on outline of the shape (Ostro \& Wisniewski 1992). The elongation of the asteroid's mean cross section was estimated to be 1.14 , measured as a ratio of the maximum to minimum breadth of the asteroid's pole-on profile (Ostro et al. 1988). The radar observations collected were only strong enough to confirm the small elongation of the asteroid and conclude that the observing geometry for the photometry was close to equatorial. The radar echo bandwidths revealed quite a large NEA with a maximum breadth of approximately $3.9 \mathrm{~km}$.

Later light curve studies refined the synodic rotation period estimate to $2.6905 \pm 0.0007 \mathrm{~h}$ (Velichko et al. 1989; Hoffmann et al. 1993; Harris 1998; Erikson et al. 2000; Manzini \& Behrend, 2013, priv. comm. ${ }^{1}$ ). A spectroscopic study from 0.8 to $2.5 \mu \mathrm{m}$ showed that the object is an Sr type in the Bus-DeMeo taxonomy, with a spectrum close to that of an ordinary chondrite meteorite rich in iron (Popescu et al. 2011). Cuyo has also been a target for rotationally resolved near-IR spectral observations with the SpeX instrument at the NASA Infrared Telescope Facility (Thomas et al. 2014). Lastly, observations from the Panoramic Survey Telescope and Rapid Response System (PanSTARRS 1) were used to determine the absolute magnitude $H=15.00 \pm 0.12$ and slope parameter $G_{12}=-0.487 \pm 0.095$ (Vereš et al. 2015).

Our observations of Cuyo were performed primarily as a part of the ESO LP and are described in Sect. 2. The wide range of observing geometries allowed us to produce a reliable shape model for the object. The shape modelling, spin-state analysis performed, and the approach taken to seek YORP-induced rotational accelerations for this object are detailed in Sect. 3. In Sect. 4, we discuss the analysis of all available spectroscopy for Cuyo, including our own optical spectra from the NTT and Palomar Observatories. We used infrared data for thermal modelling and combined it with a robust shape model obtained from convex light curve inversion to perform a thermo-physical analysis discussed in Sect. 5.

\section{Cuyo observations}

\subsection{Optical photometry campaign}

The primary source for the optical light curve observations of Cuyo within the ESO LP was the NTT, where the object was observed on 12 nights between April 2010 and April 2013, making a total of 11 light curves, as the segments collected on February 5 and 6, 2013 were folded to form a single light curve (light curve 28 in Table 1). The EFOSC2 instrument was used in imaging mode with either Bessel $V$ or $R$ filters. All the NTT images were reduced using the standard steps applicable to CCD observations. Additionally, the images taken with Bessel $R$ filter (light curves 9, 10, 13, 16, 19, 20, 21, 26, and 27, as labelled in Table 1) were fringe corrected, using a procedure similar to that described by Snodgrass \& Carry (2013).

Additional data were obtained from the ESO $2.2 \mathrm{~m}$ telescope (Chile) where four light curves were gathered from

\footnotetext{
http://obswww.unige.ch/ behrend/page_cou.html
} 
Table 1. Optical photometry data sets used in this study.

\begin{tabular}{|c|c|c|c|c|c|c|c|c|c|c|}
\hline ID & $\begin{array}{l}\text { Date } \\
\text { (UT) }\end{array}$ & $\begin{array}{c}R_{\mathrm{h}} \\
(\mathrm{AU})\end{array}$ & $\begin{array}{c}\Delta \\
(\mathrm{AU})\end{array}$ & $\begin{array}{c}\alpha \\
\left(^{\circ}\right)\end{array}$ & $\begin{array}{l}\lambda_{\mathrm{O}} \\
\left(^{\circ}\right)\end{array}$ & $\begin{array}{l}\beta_{\mathrm{O}} \\
\left(^{\circ}\right)\end{array}$ & Telescope & $\begin{array}{c}\text { Total } \\
\text { (h) }\end{array}$ & Filter & Ref. \\
\hline \multirow[t]{2}{*}{1} & 05/09/1989 & 1.193 & 0.319 & 48.5 & 309.8 & -12.4 & $\mathrm{MtL}$ & 4.1 & $V$ & 1 \\
\hline & 08/09/1989 & & & & & & $\mathrm{MtL}$ & 1.9 & $V$ & 1 \\
\hline \multirow[t]{2}{*}{2} & 07/10/1989 & 1.078 & 0.145 & 54.3 & 313.0 & -2.9 & $\mathrm{~S} 90$ & 3.7 & $V$ & 1 \\
\hline & 08/10/1989 & & & & & & S90 & 3.7 & $V$ & 1 \\
\hline 3 & 06/10/1989 & 1.080 & 0.147 & 54.0 & 312.5 & -3.1 & $\mathrm{Kh}$ & 3.7 & $V$ & 2 \\
\hline \multirow[t]{2}{*}{4} & 11/10/1989 & 1.071 & 0.143 & 56.0 & 315.2 & 0.7 & ESO1 & 1.9 & $V$ & 3 \\
\hline & $12 / 10 / 1989$ & & & & & & ESO1 & 0.5 & $V$ & 3 \\
\hline \multirow[t]{2}{*}{5} & $31 / 05 / 1995$ & 2.474 & 1.568 & 13.2 & 243.9 & -6.4 & ESOD & 4.0 & $R$ & 4 \\
\hline & 01/06/1995 & & & & & & ESOD & 5.3 & $R$ & 4 \\
\hline 6 & $02 / 08 / 2008$ & 1.215 & 0.574 & 56.3 & 33.5 & -12.9 & SAS & 1.7 & $R$ & 5 \\
\hline 7 & $05 / 08 / 2008$ & 1.199 & 0.553 & 57.4 & 36.9 & -12.3 & SAS & 2.0 & $R$ & 5 \\
\hline 8 & 07/08/2008 & 1.189 & 0.539 & 58.2 & 39.2 & -11.8 & SAS & 3.1 & $R$ & 5 \\
\hline 9 & $04 / 04 / 2010$ & 3.233 & 2.246 & 3.0 & 202.4 & -4.1 & NTT & 2.0 & $R$ & \\
\hline 10 & $06 / 04 / 2010$ & 3.233 & 2.242 & 2.7 & 201.9 & -3.0 & NTT & 3.0 & $R$ & \\
\hline 11 & 28/06/2011 & 1.815 & 0.981 & 25.2 & 285.3 & -12.6 & TMO & 3.0 & $R$ & \\
\hline 12 & $29 / 06 / 2011$ & 1.808 & 0.974 & 25.3 & 284.9 & -12.4 & TMO & 2.0 & $R$ & \\
\hline 13 & 28/08/2011 & 1.401 & 0.731 & 44.3 & 266.2 & -4.3 & NTT & 0.8 & $R$ & \\
\hline 14 & $31 / 08 / 2011$ & 1.382 & 0.723 & 45.1 & 266.6 & -4.5 & TMO & 3.0 & $R$ & \\
\hline 15 & 01/09/2011 & 1.375 & 0.721 & 45.5 & 266.8 & -4.5 & TMO & 3.0 & $R$ & \\
\hline 16 & 01/09/2011 & 1.375 & 0.721 & 45.8 & 266.8 & -4.3 & NTT & 1.2 & $R$ & \\
\hline 17 & $19 / 10 / 2011$ & 1.120 & 0.609 & 62.4 & 289.9 & -3.1 & TMO & 2.3 & $R$ & \\
\hline 18 & $20 / 10 / 2011$ & 1.116 & 0.608 & 62.7 & 290.7 & -3.0 & TMO & 2.8 & $R$ & \\
\hline 19 & $03 / 11 / 2011$ & 1.079 & 0.592 & 65.7 & 301.8 & -0.8 & NTT & 2.5 & $R$ & \\
\hline 20 & $02 / 12 / 2011$ & 1.079 & 0.621 & 64.5 & 329.7 & 4.7 & NTT & 2.5 & $R$ & \\
\hline 21 & $03 / 12 / 2011$ & 1.081 & 0.624 & 64.2 & 330.8 & 4.8 & NTT & 3.0 & $R$ & \\
\hline 22 & 02/01/2012 & 1.190 & 0.751 & 55.5 & 5.5 & 9.9 & ESO22 & 4.0 & $V$ & \\
\hline 23 & $07 / 01 / 2012$ & 1.215 & 0.780 & 53.8 & 11.5 & 10.7 & ESO22 & 1.0 & $V$ & \\
\hline 24 & 13/01/2012 & 1.248 & 0.819 & 51.9 & 18.7 & 11.7 & ESO22 & 1.0 & $V$ & \\
\hline 25 & 21/01/2012 & 1.295 & 0.876 & 49.4 & 27.9 & 13.1 & ESO22 & 1.0 & $V$ & \\
\hline 26 & $24 / 02 / 2012$ & 1.517 & 1.193 & 40.4 & 61.9 & 19.4 & NTT & 1.8 & $R$ & \\
\hline 27 & $26 / 02 / 2012$ & 1.531 & 1.216 & 40.0 & 63.6 & 19.8 & NTT & 1.7 & $R$ & \\
\hline \multirow[t]{2}{*}{28} & $05 / 02 / 2013$ & 3.113 & 2.605 & 16.9 & 204.2 & -22.0 & NTT & 1.1 & $V$ & \\
\hline & $06 / 02 / 2013$ & & & & & & NTT & 2.9 & $V$ & \\
\hline 29 & $15 / 04 / 2013$ & 3.208 & 2.229 & 5.2 & 190.4 & 7.5 & NTT & 2.0 & $V$ & \\
\hline 30 & $03 / 05 / 2013$ & 3.221 & 2.357 & 11.2 & 186.3 & 15.9 & PAL & 3.0 & $R$ & \\
\hline
\end{tabular}

Notes. For each light curve, labelled as ID 1-30, the date of the beginning of the observing night is given as well as the heliocentric $\left(R_{\mathrm{h}}\right)$ and geocentric $(\Delta)$ distances measured in $\mathrm{AU}$, the solar phase angle $(\alpha)$, and the observed ecliptic longitude $\left(\lambda_{\mathrm{O}}\right)$, and latitude $\left(\beta_{\mathrm{O}}\right)$ of the target. In some cases (light curves 1,2,4,5, and 28) a single light curve has been composed of segments gathered over a few nights. The designation of instrument used is listed in the "Telescope" column. For each light curve segment a total time span of the light curve in hours, and imaging filter used are listed in columns "Total" and "Filter", respectively. Where applicable, a reference to the already published work is given in the "Ref." column. Telescope key (with MPC site code): (MtL, I52) NASA-UA Mt. Lemmon 60 in, Arizona, USA; (S90, 695), Steward Observatory 90 in Bok Telescope, Arizona, USA; (Kh, 121), Kharkiv Astronomical Observatory 0.7 m Telescope, Ukraine; (ESO1, 809), European Southern Observatory (ESO) $1 \mathrm{~m}$ Telescope, La Silla, Chile; (ESOD, 809), Danish $1.54 \mathrm{~m}$ Telescope, La Silla, Chile; (SAS, A12), Stazione Astronomica di Sozzago 0.4 m Telescope, Italy; (NTT, 809), European Southern Observatory $3.5 \mathrm{~m}$ New Technology Telescope, Chile; (TMO, 673), Table Mountain Observatory, California, USA; (ESO22, 809), ESO/Max Planck Institute $2.2 \mathrm{~m}$ Telescope, La Silla, Chile; (PAL, 675), Palomar Observatory $5 \mathrm{~m}$ Hale Telescope, California, USA.

References. (1) Wisniewski et al. (1997); (2) Velichko et al. (1989); (3) Hoffmann et al. (1993); (4) Erikson et al. (2000); (5) Beherend \& Manzini (priv. comm.).

February 2-21, 2012. The ESO $2.2 \mathrm{~m}$ telescope at La Silla is equipped with a Wide Field Imager instrument where data is simultaneously collected on eight CCD chips, making a mosaic image. Before performing any reduction, the chip collecting the object images was identified and any data reduction was performed only on that chip using standard steps.

Further light curve data were collected at two other facilities, including the JPL $0.6 \mathrm{~m}$ telescope at Table Mountain
Observatory (USA), where six light curves were collected, and at the Palomar $5 \mathrm{~m}$ (USA) where we obtained a single light curve. There was no special treatment required for the Table Mountain Observatory and Palomar images other than the standard CCD reduction procedures.

The details of the ESO LP optical light curve campaign along with all other available light curve data are gathered in Table 1. The observational circumstances are illustrated in Fig. 1. A wide 

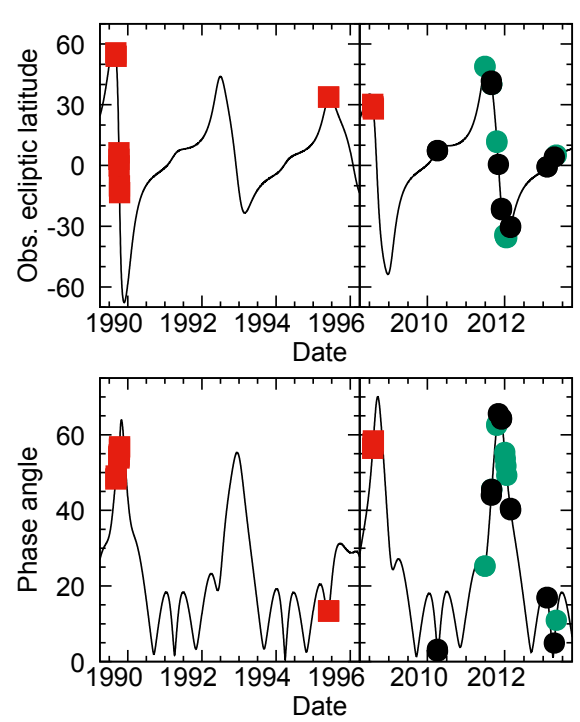
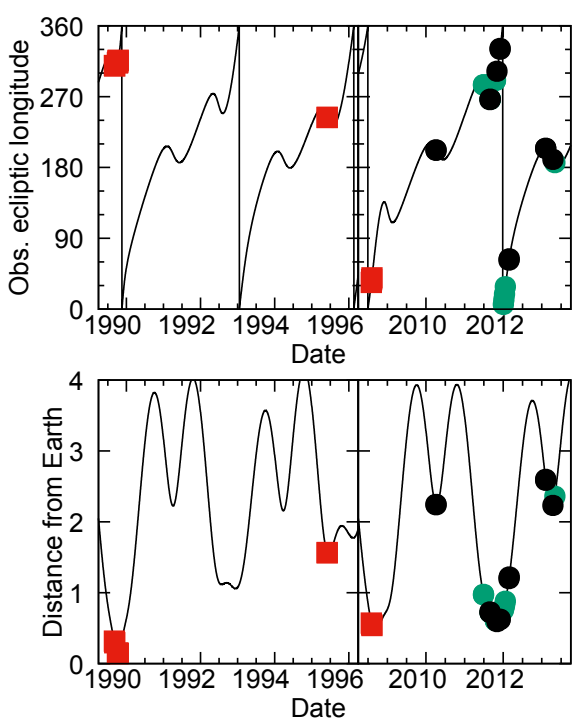

Fig. 1. Asteroid Cuyo observing geometries during the light curve observations. The graphs display different quantities as a function of time (in years). Top two panels: positions of the object in the ecliptic coordinate system, latitude and longitude, as observed from Earth. Bottom two panels: phase angle (angle between the positions of Earth and Sun as observed from the target), and geocentric distance to the target. Optical light curve data from NTT are marked with filled black circles, with light curve data from supporting campaigns marked with filled green circles. Red squares represent the additional light curve data available. The black continuous line is the object's observational ephemeris. range of observing geometries covered enables a robust shape and pole determination.

Photometry was performed on all of the imaging data collected with the ESO LP and associated programmes. The imaging observations of Cuyo were acquired in several ways. When the asteroid was sufficiently bright and slow moving, we reduced the exposure times so that the asteroid moved well within the seeing disc during a single image and tracked at sidereal rates. This allowed for the full recovery of the Point Spread Function (PSF) for both background stars and asteroid, and thus more accurate photometry, especially when several frames were stacked. When this could not be done we simply tracked at asteroid rates of motion, and ascertained the frame PSF from the asteroid itself. We extracted the rotational light curves using relative photometry, that is by comparing the brightness of the asteroid with that of a set of non-varying background stars. Standard aperture photometry techniques were applied, but we let the aperture size vary according to the seeing value, that is we kept the aperture size a fixed multiple of the Full Width Half Maximum (FWHM) of the frame PSF. The optimal balance between having enough signal from the asteroid within the aperture and excluding as much noisy sky as possible is achieved using aperture sizes of around $2 \times F W H M$. All of the extracted light curves are presented in the appendix (Fig. A.1) and Table A.1.

The previously published data on Cuyo include eight light curves (see Table 1). The data sets from Mt. Lemmon and Kitt Peak, dating back to September and October 1989, contain two composite light curves (Wisniewski et al. 1997). Another light curve was taken on one night at the Kharkiv Astronomical Observatory on October 6, 1989 (Velichko et al. 1989). The observations with the ESO $1 \mathrm{~m}$ telescope, spanning four nights, had been folded to produce two light curves (Hoffmann et al. 1993; presented in their Figs. 1 and 2). However, just one of them could be reliably linked to other data, and the other showed a very different periodicity to that of Cuyo, so was likely erroneously labelled as Cuyo and was not used in our analysis. The data from 1995 observations at the ESO Danish $1.5 \mathrm{~m}$ were taken across two nights (see Erikson et al. 2000, Fig. 2). The data set from the Stazione Astronomica di Sozzago $0.4 \mathrm{~m}$ telescope, gathered in 2008, was provided by the observers. Those light curves can be viewed online (Manzini \& Behrend 2013, priv. comm. ${ }^{1}$ ).

\subsection{Spectroscopic observations}

NTT - July 25, 2011. We obtained low resolution, longslit CCD spectra of Cuyo on the night of July 25, 2011 (UT) at the NTT. We used the EFOSC2 instrument in spectroscopy mode (Buzzoni et al. 1984). The camera employed a $2048 \times 2048$ pixel CCD for spectroscopy, which we used in $2 \times 2$ binning mode. To optimize spectral resolution and wavelength coverage, we observed with the Grism\#1 (100 lines per mm grating). This setup gave us useful coverage from $0.4-1.0 \mu \mathrm{m}$ with a dispersion of $\sim 13.3 \AA \mathrm{pixel}^{-1}$. All asteroid observations were made with a $2^{\prime \prime}$ wide slit, aligned at the parallactic angle to minimise any adverse affects of atmospheric dispersion, which can lead to confounding curvature in the extracted spectrum thus potentially corrupting any derived taxonomic classification. Wavelength calibrations were performed using He-Ar arc-lamp spectra obtained with the same instrumental set-up, which provided a sufficient number of well-defined emission lines across the entire wavelength range considered. We obtained a total of three spectra of Cuyo, at a single rotational phase. Each spectrum had an exposure time of $200 \mathrm{~s}$ and the airmasses were $\sim 1.8$. We also obtained exposures of a solar analogue star at airmasses matching that of the asteroid. Sky conditions during the spectral observations were photometric.

Each spectral image was reduced separately in the following manner. After flat-fielding, bias subtraction, and cosmic-ray removal, the flux from the object of interest was isolated by summing the counts along the spatial axis within an aperture centred on the brightest pixel in the slit, and wide enough to take in all light from the asteroid or solar analogue star. This allowed us to collapse the images along the spatial dimension into one-dimensional spectra. Night-sky background spectra were extracted in the same way, but with 20-pixel-wide sky apertures set immediately adjacent to the object aperture. The average skybackground spectrum was then simply subtracted from the object spectrum. The solar analogue stars were reduced in an identical way, with solar analogue spectra paired to asteroid spectra according to airmass. By dividing the raw spectrum of Cuyo with that of the solar analogue, we obtained our reduced plot of spectral reflectance. We note that the asteroid and solar analogue stars were positioned on the same part of the slit and the "aperture response function" or "slit illumination" was flat within the spatial extent of all object and sky apertures. 
NTT - December 4, 2011. We obtained additional low resolution long-slit CCD spectra of Cuyo on the night of December 4, 2011 (UT) at the NTT. The instrumental set-up and observational approach was identical to the July 25 observations, including the choice of arc-lamp spectra, for wavelength calibration. We obtained a total of six spectra of Cuyo, over a single $2.7 \mathrm{~h}$ period, one full rotation of the asteroid. Each spectrum had an exposure time of $90 \mathrm{~s}$ and the airmasses were $<1.8$. We also obtained exposures of a solar analogue star at airmasses matching that of the asteroid. Sky conditions during the spectral observations were photometric.

Palomar - February 2, 2012. We obtained low resolution long-slit CCD spectra of Cuyo on the night of February 2, 2012 (UT) using the $5.1 \mathrm{~m}$ Hale Telescope at Palomar Observatory with the facility Double Spectrograph mounted at the cassegrain focus (Oke \& Gunn 1982). This instrument utilises a dichroic beam-splitter, which directs light to blue and red grating spectrometers equipped with separate CCD cameras. Each camera employed a $1024 \times 1024$ pixel CCD. To optimise spectral resolution and wavelength coverage, we observed with the D52 dichroic (red/blue transition at $520 \mathrm{~nm}$ ) and the 300 line per $\mathrm{mm}$ grating for the blue spectrometer and the 316 line per $\mathrm{mm}$ grating for the red spectrometer. This set-up effectively gave us continuous coverage from $0.3-1.0 \mu \mathrm{m}$ with a dispersions of 3.39 and $4.89 \AA$ per channel for the blue and red cameras, respectively. All observations were made with a 6 " wide slit, aligned in the default north-south orientation. Wavelength calibrations were performed using arc-lamp spectra obtained before evening twilight. We also obtained exposures of a solar analogue star at airmasses bracketing that of the asteroid.

\subsection{Thermal-infrared observations with ESO VLT VISIR}

The optical observations were supplemented with thermalinfrared photometry from the VISIR instrument (Lagage et al. 2004) on Unit 3 "Melipal" of the $8.2 \mathrm{~m}$ Very Large Telescope (VLT) array at ESO's Paranal Observatory in Chile. In particular, Cuyo was observed three times on September 5, December 17, and December 19, 2011 (see Table 2 for a summary of the observational circumstances) in imaging mode with VISIR's intermediate field, which had a pixel scale of $0.127 \mathrm{arcsec} \mathrm{pixel}^{-1}$ and a total field of view of $32.5 \operatorname{arcsec} \times 32.5 \operatorname{arcsec}$. Integration times were chosen to give a minimum signal-to-noise ratio (S/R) of 10 in each filter, and the observations were chop-nodded with perpendicular throws of 8 arcsec for sky-background subtraction. For calibration purposes, suitable standard stars that were within $2 \mathrm{~h}$ RA of Cuyo were selected from Cohen et al. (1999), and were observed at airmasses that covered a similar range to Cuyo (i.e. 1.0-2.0). To produce the flux measurements, the nod pair images were combined and reduced using the VISIR pipeline (Pantin et al. 2008), and photometry was performed on the combined images using aperture radii of $3,5,7$, and 10 pixels with a background annulus of 15-20 pixels (Wolters et al. 2011; Duddy et al. 2012). An atmospheric extinction correction ( 5\%) and an aperture correction $(\sim 10 \%)$ was applied based on the calibration obtained from the standard stars. The calibrated fluxes measured through each aperture radius were consistent with one another, and the five pixel aperture radius was selected as it gave the highest $\mathrm{S} / \mathrm{N}$ after combining, in quadrature, the uncertainties from photon statistics, extinction correction, and aperture correction. Finally, the flux from all four beams in each combined image was summed (i.e. the negative beams were multiplied by -1)
Table 2. Observational circumstances of Cuyo during the ESO VLT observations with VISIR.

\begin{tabular}{cccccc}
\hline \hline Date & $\begin{array}{c}\text { Total } \\
(\mathrm{h})\end{array}$ & $\begin{array}{c}\text { Wavelength range } \\
(\mu \mathrm{m})\end{array}$ & $\begin{array}{c}R_{\mathrm{h}} \\
(\mathrm{AU})\end{array}$ & $\begin{array}{c}\Delta \\
(\mathrm{AU})\end{array}$ & $\begin{array}{c}\alpha \\
\left(^{\circ}\right)\end{array}$ \\
\hline $05 / 09 / 11$ & 2.2 & $8.59-12.47$ & 1.349 & 0.711 & 47.0 \\
$17 / 12 / 11$ & 3.0 & $8.59-12.47$ & 1.121 & 0.672 & 60.6 \\
$19 / 12 / 11$ & 1.1 & $8.70-18.72$ & 1.128 & 0.681 & 60.0 \\
\hline
\end{tabular}

Notes. For each set of infrared observations, labelled with the "Date" of the beginning of the observing night the "Total" number of hours observed is given as well as the "Wavelength range" in $\mu \mathrm{m}$, the heliocentric $\left(R_{\mathrm{h}}\right)$ and geocentric $(\Delta)$ distances measured in $\mathrm{AU}$, and the solar phase angle $(\alpha)$.

to give the total measured flux, and these fluxes are provided in Table A.2.

\section{Shape modelling with optical light curve data, and searching for YORP-induced rotational accelerations}

We developed shape modelling procedures that use the inversion methods described by Kaasalainen \& Torppa (2001), Kaasalainen et al. (2001), and Durech et al. (2010). The asteroid shapes we obtain in this study are all convex shapes, which means they approximate the real asteroid shapes, but without any surface concavities. To ensure the best results from our shape and spin-state modelling, we included all available light curves in the modelling, which meant using data from a long time span for a YORP-detection candidate. We have modified the shape modelling procedure to accommodate the possible YORP-induced change in rotation period parallel with developing the shape model.

The shape modelling and spin-state analysis for the purpose of YORP detection involves investigating the timing of the light curve observations. The light curves can be represented in time domain or in rotation phase, $\varphi$, when the rotation period, $P$, is known. Accounting for the linear change of rotation rate, $\omega$, with time, the rotation phase of an asteroid can be expressed for any given time as

$\varphi(t)=\varphi\left(T_{0}\right)+\omega\left(t-T_{0}\right)+\frac{1}{2} v\left(t-T_{0}\right)^{2}$,

where:

$\varphi(t) \quad$ observed rotation phase in radians,

$t \quad$ the time of observation (JD),

$\varphi\left(T_{0}\right) \quad$ initial rotation phase in radians,

$T_{0} \quad$ epoch of the model from which the model is propagated (JD),

$\omega \quad$ rotation rate in $\operatorname{rad~day~}^{-1} ; \omega \equiv 2 \pi / P, P$ is rotation period in days,

$v \quad$ the change of rotation rate in $\operatorname{rad~day~}^{-2} ; v \equiv \dot{\omega}$ (i.e. the observed YORP strength).

The linear change of rotation rate, $v$, can be attributed to the spin component of the YORP torque (Rubincam 2000), hereafter referred to as the YORP factor, or YORP strength.

The initial step in the shape modelling is to obtain an estimate of the sidereal rotation period. The rotation period is then refined at various stages of the shape and spin-state modelling, 


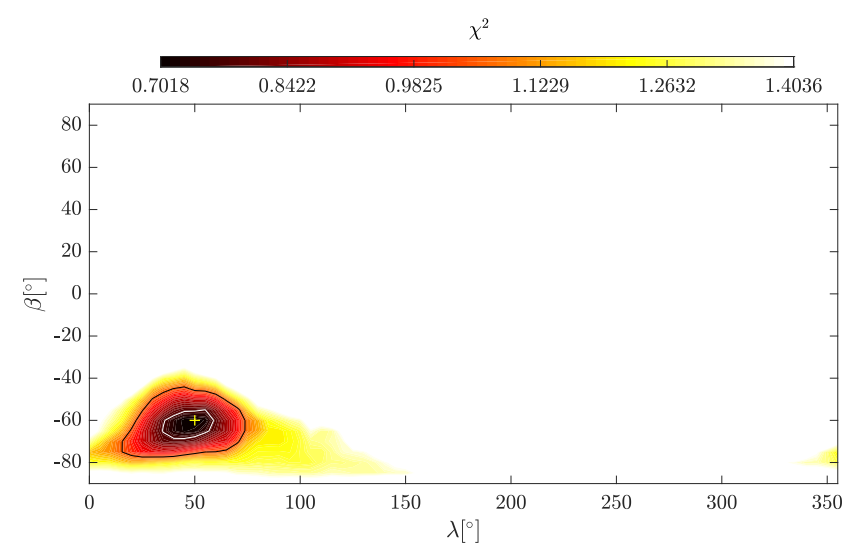

Fig. 2. Goodness-of-fit $\chi^{2}$ plane for the pole scan for (1917) Cuyo with a constant rotation period. For a $5^{\circ} \times 5^{\circ}$ grid of possible pole positions, in ecliptic longitude $\lambda$ and latitude $\beta$, the shape model and sidereal rotation period were optimised. Goodness-of-fit $\chi^{2}$ values for each model were recorded. They are colour coded, with darker colours marking the lower $\chi^{2}$ values and anything above twice the minimum value of $\chi^{2}$ is white. The white contour line indicates a $10 \%$ increase relative to the minimum $\chi^{2}$ value obtained (marked with a " + ") and the black contour line is a $50 \%$ increase. The fragment of the celestial sphere was chosen with the smallest $\chi^{2}$ values, for a more detailed $1^{\circ} \times 1^{\circ}$ scan, as shown in Fig. 3 .

but a good starting point is essential. For Cuyo we used the synodic rotation period of $2.6905 \mathrm{~h}$ (Wisniewski et al. 1997). This was later updated through fits of the available light curve data using a simplified shape model and a crude selection of possible pole positions. An initial value of the sidereal rotation period of $2.6897651 \mathrm{~h}$ was adopted for the shape modelling procedures, but it was further refined as one of the fitted parameters. The $T_{0}$ parameter was fixed to be 2456416.0 (Mar. 3, 2013), which is close to the last observation.

The whole celestial sphere was initially sampled with a $5^{\circ} \times 5^{\circ}$ resolution in ecliptic longitude, $\lambda$, and latitude, $\beta$. At each point on the grid a convex shape model and sidereal rotation period were optimised while keeping the pole position fixed. Goodness-of-fit $\chi^{2}$ values, for fitting the model to the light curve data, were recorded to create a $\chi^{2}$-plane, as illustrated in Fig. 2.

After this initial scan was finished the search for the pole position was then narrowed down to a region of the celestial sphere with the lowest $\chi^{2}$ and a refined $1^{\circ} \times 1^{\circ}$ scan was performed with results presented in Fig. 3. Such $\chi^{2}$-planes on a fragment of celestial sphere were created for a range of YORP factor values with increments of $10^{-9} \mathrm{rad} \mathrm{day}^{-2}$ between $-2 \times 10^{-8}$ and $2 \times 10^{-8}$ rad day $^{-2}$, and at coarser steps of $5 \times 10^{-9}$ rad day $^{-2}$ between $-10^{-7}$ and $10^{-7}$ rad day $^{-2}$.

The best-fit models from each $\chi^{2}$-plane were then extracted and examined. The smallest $\chi^{2}$ value from each YORP plane has been plotted against the YORP factor corresponding to that plane to identify the best solution and assess the possibility of a YORP detection (see Fig. 4). While the best-fit solution is at $v=10^{-9} \mathrm{rad} \mathrm{d}^{-2}$, the 0 -YORP solution has only $0.01 \%$ worse $\chi^{2}$, so it is the latter that is adopted here as the nominal model (see Table 3). The broad minimum of the $\chi^{2}$ plot includes noYORP (constant rotation period), as well as positive and negative rotation rate change solutions. All of those lay within $1 \%$ above the minimum $\chi^{2}$ value. The three cases are indistinguishable, each producing models that can perfectly reproduce the shapes of light curves from all the epochs. For both the spin-up and slow-down scenarios one example light curve fit is shown in

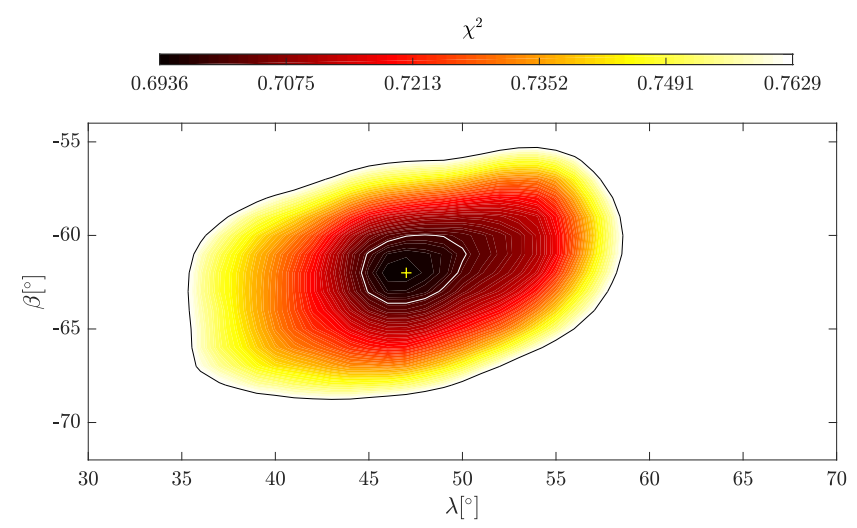

Fig. 3. Same as Fig. 2 but for a $1^{\circ} \times 1^{\circ}$ grid of possible pole positions, covering just a fragment of the celestial sphere around the best solution, $\lambda \in\left(30^{\circ}, 70^{\circ}\right), \beta \in\left(-72^{\circ},-54^{\circ}\right)$. Goodness-of-fit $\chi^{2}$ values are colour coded, with darker colours marking the lower $\chi^{2}$ values and anything above a $10 \%$ increase above the minimum $\chi^{2}$ value is white. The yellow contour line indicates a $1 \%$ increase relative to the minimum $\chi^{2}$ value obtained (marked with a “+”); the black contour line is a $10 \%$ increase.
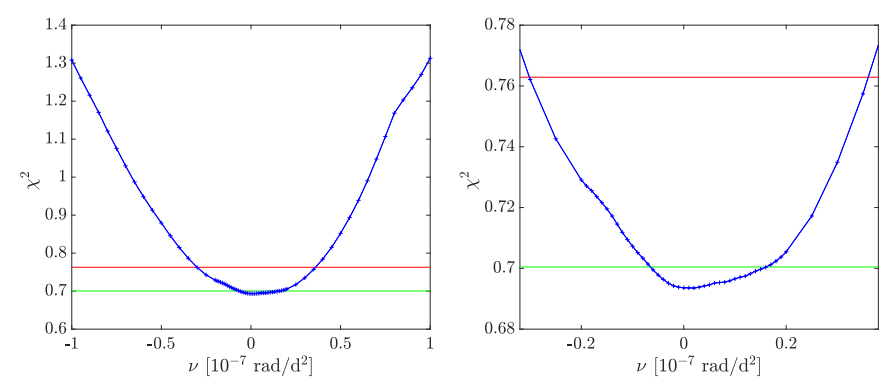

Fig. 4. YORP-factor fit to the light curve data. For each probed value of possible YORP-induced spin-up factor, $v$, marked on the horizontal axis, a $\chi^{2}$-plane was constructed on a fragment of the celestial sphere, similar to the 0-YORP plane shown in Fig. 3. Minimum $\chi^{2}$ value, corresponding to the best solution from each plane, versus YORP factor are plotted with blue crosses. The horizontal lines mark the increases above the best-fit $\chi^{2}$ minimum: $+1 \%$ (green) and $+10 \%$ (red). A zoomed view is provided in the right panel and shows that there is a wide range of possible solutions including spin-up, spin-down, or no rotation period change.

Fig. 5. Even though in both plots the model has been propagated with a starting point, $T_{0}$, set in 2013 and each has a different starting rotation period $\left(P_{0}\right.$ is the sidereal rotation period at epoch $T_{0}$, as the initial rotation period will change due to YORP) and YORP factor, they both reproduce the light curve taken in 1989.

Shape models, which can reproduce all $24 \mathrm{yr}$ of light curve observations, can be derived for a constant rotation period solution just as well as for YORP strengths within the limits determined, $(-0.7$ to 1.7$) \times 10^{-8} \mathrm{rad} \mathrm{day}^{-2}$. The nominal constant-period model has a slightly flattened shape and a "quasi-triangular" outline when viewed from the pole-on direction as shown in Fig. 6. The profile of the asteroid is consistent with the mean cross sections obtained from early convex-profile light curve inversion performed for Cuyo (Ostro \& Wisniewski 1992, Fig. 1). The model seems to be physically feasible, it has the spin-axis aligned with the maximum moment of inertia, and is not excessively flattened or elongated. Additionally, the shape appears to have an equatorial bulge, which might suggest surface material arrangement as a result of fast rotation. 

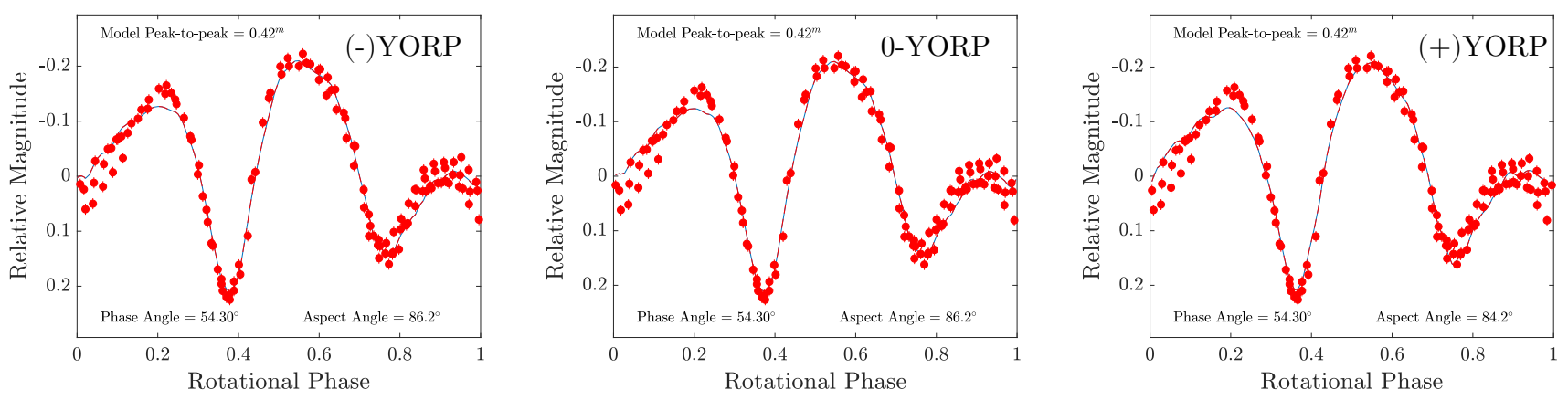

Fig. 5. Light curve fits to best-fit models from selected YORP planes. All models have the same starting epoch, $T_{0}=2456416.0$. The example light curve was taken at $T_{0}=22 \mathrm{yr}$ (Wisniewski et al. 1997, labelled "ID 2" in Table 1). All models have been developed using the same light curve set as the nominal best-fit model (see Table 3). Left panel: YORP-induced slow-down model, with $v=-0.07 \times 10^{-7} \mathrm{rad} \mathrm{day}^{-2}$ and rotation pole $\lambda=47^{\circ}, \beta=-62^{\circ}$. Middle panel: nominal model, with $v=0$ rad day $^{-2}$ and rotation pole $\lambda=47^{\circ}, \beta=-62^{\circ}$. Right panel: model showing a YORP-induced spin-up, with $v=0.17 \times 10^{-7}$ rad day $^{-2}$ and rotation pole $\lambda=51^{\circ}, \beta=-61^{\circ}$. The quality of the light curve fit for all three models is very close.

Table 3. Summary of derived model parameters for (1917) Cuyo.

\begin{tabular}{ll}
\hline \hline$\lambda$ & $47^{\circ} \pm 11^{\circ}$ \\
$\beta$ & $-62^{\circ} \pm 6^{\circ}$ \\
$T_{0}(\mathrm{JD})$ & 2456416.0 \\
$P(\mathrm{~h})$ & $2.6897642 \pm 0.000004$ \\
$\nu\left(\times 10^{-7} \mathrm{rad} \mathrm{d}^{-2}\right)$ & $0_{-0.07}^{+0.17}$ \\
\hline
\end{tabular}

Notes. The presented set of parameters corresponds to the best-fit constant-period solution. The table lists: the ecliptic coordinates of the rotation pole, longitude $(\lambda)$ and latitude $(\beta)$, the model epoch according to convex inversion output $\left(T_{0}\right.$, set at the end of 2013), the sidereal rotation period $(P)$, and the YORP spin-up factor $(v)$, which is 0 for the nominal constant-period solution, with the uncertainties showing the range of possible values within the $1 \%$ increase of the $\chi^{2}$ value for the best-fit solution.

\section{Analysis of spectroscopic observations}

For each set of optical spectra that we acquired (normalised at $5500 \AA$ ) we performed a classification analysis based on the BusDeMeo taxonomy (Bus \& Binzel 2002; DeMeo et al. 2009). Each spectrum was resampled at the wavelengths used in the Bus-DeMeo taxonomy and the normalised reflectances compared directly using a standard $\chi^{2}$ minimisation process. Figure 7 illustrates the results for the NTT spectra obtained on the evening of July 25, 2011. All three spectra are very consistent with each other and the mean spectrum of Sv types from the Bus-DeMeo taxonomy. On this occasion we sampled at effectively just one rotation phase. Table 4 provides the observing geometry on this date (see IDs 11-13), including the asteroid-centric coordinates of the sub-observer point.

We returned to this object on December 4, 2011 with the same instrument, with the aim of following the asteroid across one full rotation. The extracted spectra are shown in Fig. 8. Again, the spectra are very consistent with each other, and range from $\mathrm{S}$ to $\mathrm{Sv}$ types. While there appears to be a correlation of type with rotation phase (see Table 4), given the similarity of these two types at optical wavelengths and the $\mathrm{S} / \mathrm{N}$ attained, we do not assign much weight to this observation.

Asteroid Cuyo has been observed by others, not just at optical wavelengths but also in the near-IR $(1.0-2.5 \mu \mathrm{m})$. On the optical side, a spectrum acquired at the McGraw-Hill 2.4-m telescope was classed as Sl type (Binzel et al. 2004) in the
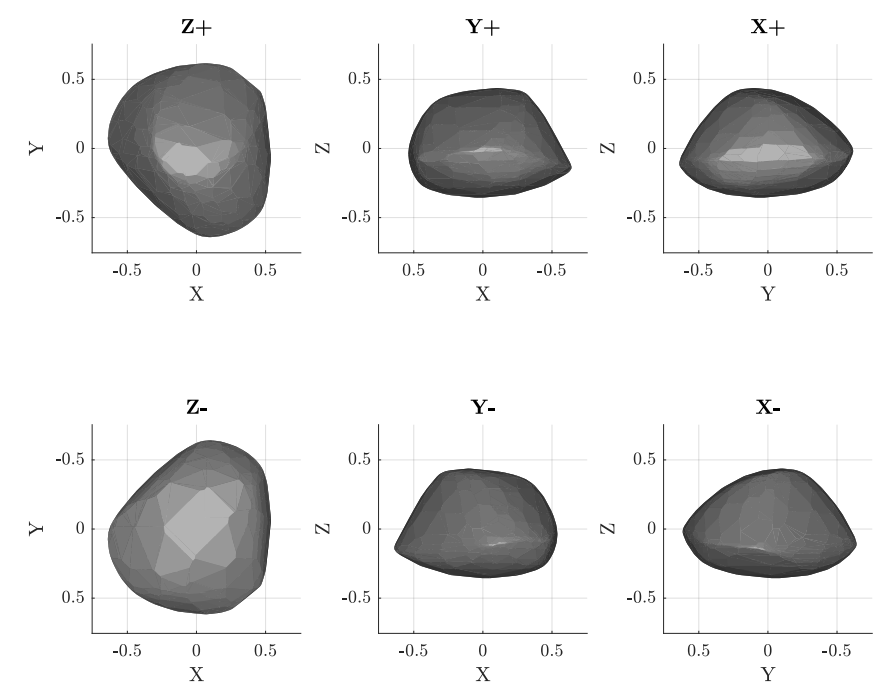

Fig. 6. Best-fit shape model for asteroid Cuyo. The shape model was derived from the best-fit pole solutions, assuming a constant rotation period. Top row, left to right: views along the $Z, Y$, and $X$ axes of the body-centric coordinate frame from the positive end of the axis. Bottom row, left to right: views along the $Z, Y$, and $X$ axes from their negative ends. While the $Z$-axis of the body (also the spin axis) is aligned with the shortest axis of inertia, there is no relation between the longest axis of inertia and the $X$-axis of the body, as used in shape modelling and spin-state analysis. The $X$-axis is arbitrarily selected so that it would be in the plane of sky at $T_{0}$.

Bus taxonomy (Bus 1999). Similarly, the NTT was utilised to obtain an optical spectrum in January 2003, and subsequently classed as S type (Michelsen et al. 2006) using the Tholen taxonomy (Tholen 1984). For consistency, we re-analysed all of the previously-published spectra and classed each one within the Bus-DeMeo taxonomy, using precisely the same method as above. The results of this are presented in Table 4 (the original classifications are given in parentheses).

Spectra that extend well into the near-IR are much more diagnostic for classification purposes. A single near-IR spectrum (covering 0.8-2.5 $\mu \mathrm{m}$ ) was classified as Sr type in the Bus-DeMeo taxonomy, with a spectrum close to that of an ordinary chondrite meteorite rich in iron (Popescu et al. 2011). However, the spectrum was difficult to classify robustly, with several silicate-rich asteroid types being possible, yet none fitting the shape of the spectrum well. Our re-analysis of the 

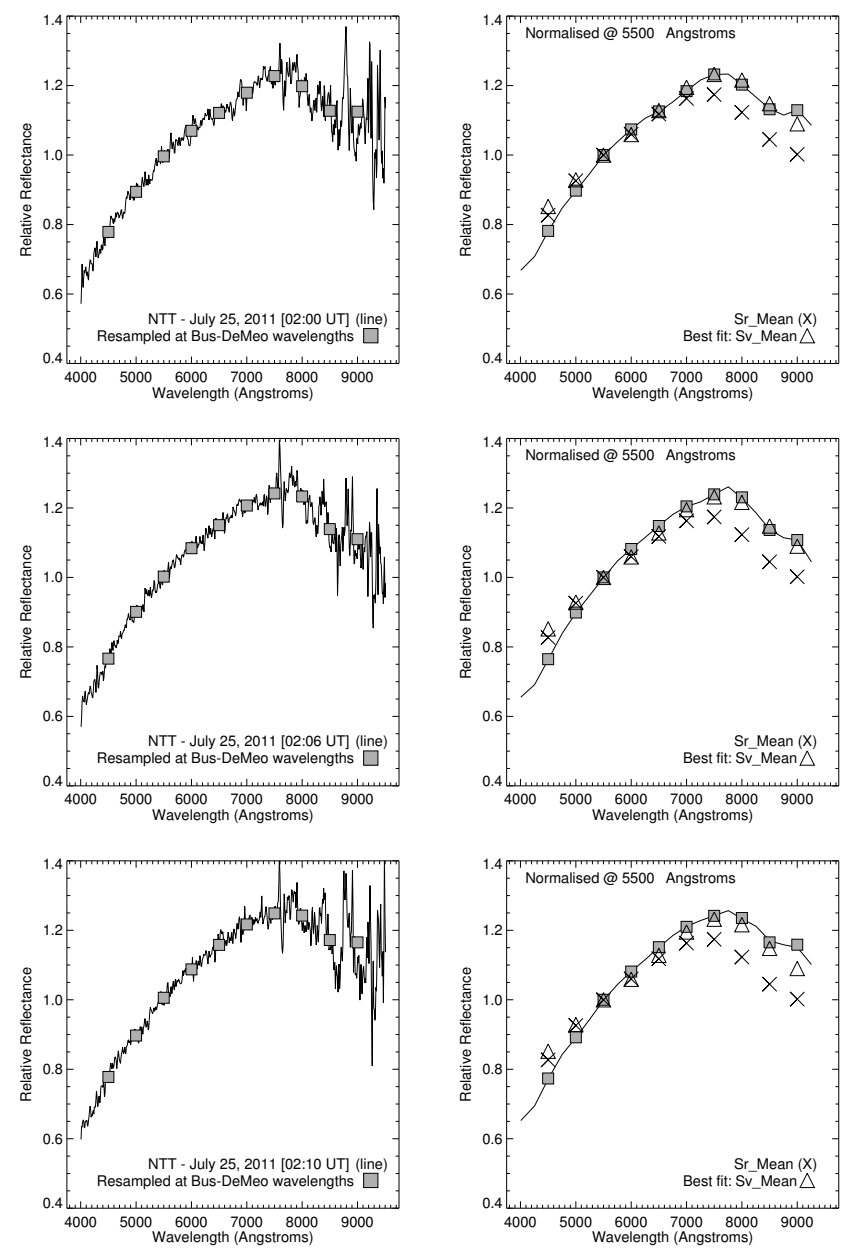

Fig. 7. Spectroscopy of (1917) Cuyo. The spectra were collected on July 25, 2011 with the ESO NTT telescope. All spectra are normalized to unity at $5500 \AA$. The panels on the left show the raw extracted spectra, and a binned version overplotted. The panels on the right include the NTT spectra resampled at the wavelengths used in the Bus-DeMeo taxonomy (Bus \& Binzel 2002; DeMeo et al. 2009) and compared with those classes that most closely match Cuyo. The spectra are quite consistent with either S or Sv types, and no robust indication of compositional variations is evident. We also include the mean spectrum for the $\mathrm{Sr}$ type from the Bus-DeMeo taxonomy, just to highlight how distinctive this class is from $\mathrm{S} / \mathrm{Sv}$ types, even within this wavelength range. Table 4 gives additional information related to the observing geometry on this date (IDs 11-13).

Popescu et al. (2011) spectrum shows R type to be the best match (see Fig. 9).

Time series near-IR $(\sim 0.7-2.5 \mu \mathrm{m})$ spectra were acquired using the SpeX instrument on the NASA Infrared Telescope Facility (IRTF; Thomas et al. 2014). The object was observed six times on June 24, 2011, over a period of time covering a little more than three hours, and an additional spectrum was acquired on August 14, 2011. The time span of observations covered a bit more than one full rotation to probe different areas of the asteroid's surface. The authors found that either Sv or Sr fit the spectra best. Analysis of the location of the $1 \mu \mathrm{m}$ spectral feature (band I Centre) led the authors to conclude that no significant variation in surface composition is evident on Cuyo. We re-analysed these spectra, and while we agree with this conclusion, there still appear to be significant differences in the spectra with rotation when the full wavelength range is considered. Figure 10 shows our spectral classifications. We also find that $\mathrm{Sv}$ or Sr fit the spectra best, but not necessarily in the same order as Thomas et al. (2014) There may be indications of spectral differences with rotation from these data. In spectrum " $A$ " the asteroid lines up very well with Sr type, but from spectra "B" to "E" the spectra are significantly different and are a close match to Sv types (spectrum " $F$ " is ambiguous in this respect and lies between the two classes). The Sr spectrum ("A") is not repeated roughly one full rotation later (spectrum "E"), but there is still a noticeable difference in rotation phase between the two cases, which may account for this $\left(\sim 20^{\circ}\right)$. Spectra "B" and "F" are a much closer match in terms of observing geometry though, but the distinction between the two spectra at these times is less clear.

These observations are more clearly seen in Fig. 12, where we plot the observational geometry of all the spectra obtained on this object to date, including ours (also see Table 4 to link the spectrum IDs with UT or previous observer). We consider the high latitudes first (i.e. $>+50^{\circ}$ latitude: IDs 5-10, 11-14). There may be indications of longitudinal variation in surface composition with Sr-type material residing around $165^{\circ}$ longitude. At other observed positions we measure an Sv spectral type (the spectra corresponding to IDs 10 and 14 are ambiguous). However, the origin of these different taxonomic types is currently uncertain. We can speculate that $\mathrm{Sr}$ material may be highly weathered due to the large optical slope, but that would not explain why the $1 \mu \mathrm{m}$ absorption is so deep. Similarly, Sv may indicate a contribution from basaltic V-type material to weathered S-type material on the surface, but it is unclear why this would be mixed with $\mathrm{Sr}$ material.

Spectra at mid-range latitudes (i.e. $+30^{\circ}$ to $-30^{\circ}$ latitude, IDs 1-4, 15-20) show no coherence at all, when considered collectively. However, if we consider just the near-IR spectra from this set (IDs 3 and 4), which cover a more diagnostic range of wavelengths, there may be signs of compositional variation at these equatorial latitudes (see Figs. 9 and 12). Spectra taken at visible wavelengths only are predominantly $\mathrm{S}$ or Sv types.

Finally, our Palomar Observatory optical spectrum, acquired on February 2, 2012, was fortuitously acquired when the subEarth point was at a high southern latitude, effectively allowing the objects' southern pole region to be sampled. The spectrum was classified as Sq type but Sr type is also a close match, which are both quite distinctive from the spectra of $\mathrm{S}$ or Sv types (see Fig. 11). Unfortunately, the spectrum signal to noise was quite low and given the restrictive wavelength range of the spectrum is it quite difficult to draw any solid conclusions about compositional differences between, say, the south pole and equatorial regions. Overall, we find that the prospect of surface compositional variations, as surmised from the complete set of data, certainly warrants additional follow-up study.

\section{Thermal modelling of ESO VLT VISIR data}

The ESO VLT VISIR observations were modelled using the Near-Earth Asteroid Thermal Model (NEATM, Harris 1998) and with the more sophisticated Advanced Thermo-Physical Model (ATPM, Rozitis \& Green 2011, 2012, 2013) to determine the size and surface properties of Cuyo, and to also provide a theoretical prediction of its YORP rotational acceleration.

\subsection{Results for the Near-Earth Asteroid Thermal Model (NEATM)}

Simultaneous measurements of the asteroid flux in the visible and in the thermal-infrared allow both the diameter and 
Table 4. Cuyo optical and near-infrared spectra.

\begin{tabular}{|c|c|c|c|c|c|c|c|c|c|c|}
\hline ID & Date & UT & $\begin{array}{l}\text { Aspect } \\
\left(^{\circ}\right)\end{array}$ & $\begin{array}{l}\alpha \\
\left({ }^{\circ}\right)\end{array}$ & $\begin{array}{l}\text { Lat. } \\
\left({ }^{\circ}\right)\end{array}$ & $\begin{array}{l}\text { Lon. } \\
\left({ }^{\circ}\right)\end{array}$ & $\begin{array}{l}\text { Spec. } \\
\text { class }\end{array}$ & $\begin{array}{l}\text { Wavelength } \\
\text { range }\end{array}$ & Facility & Ref. \\
\hline 1 & 01-Apr.-1994 & & 67.2 & 0.6 & 22.8 & & Sv/S (Sl) & VIS & MDM & 1 \\
\hline 2 & 03-Jan.-2003 & $08: 41$ & 90.8 & 24.8 & -0.8 & 129.0 & $\mathrm{~S} / \mathrm{Sv} / \mathrm{Sr}$ & VIS & NTTa & 2 \\
\hline 3 & 07-Jul.-2008 & $14: 13$ & 77.6 & 47.6 & 12.4 & 75.0 & $\mathrm{~S} / \mathrm{Sv}(\mathrm{Sv})$ & NIR & IRTF & $4^{(a)}$ \\
\hline 4 & 27-Aug.-2008 & $15: 17$ & 104.3 & 66.0 & -14.3 & 214.3 & $\mathrm{R}(\mathrm{Sr})$ & NIR & IRTF & 3 \\
\hline 5 & 24-Jun.-2011 & $10: 16$ & 35.4 & 24.8 & 54.6 & 164.5 & $\mathrm{Sr}(\mathrm{Sv})$ & NIR & IRTF & 4 \\
\hline 6 & 24-Jun.-2011 & $10: 46$ & 35.4 & 24.8 & 54.6 & 97.6 & $\mathrm{~Sv}(\mathrm{Sr})$ & NIR & IRTF & 4 \\
\hline 7 & 24-Jun.-2011 & $11: 31$ & 35.4 & 24.8 & 54.6 & 357.2 & $\mathrm{~Sv}(\mathrm{~Sv} / \mathrm{Sr})$ & NIR & IRTF & 4 \\
\hline 8 & 24-Jun.-2011 & $12: 11$ & 35.4 & 24.8 & 54.6 & 268.0 & $\mathrm{~Sv}(\mathrm{Sr})$ & NIR & IRTF & 4 \\
\hline 9 & 24-Jun.-2011 & $12: 48$ & 35.4 & 24.8 & 54.6 & 185.4 & $\mathrm{~Sv}(\mathrm{~Sv} / \mathrm{Sr})$ & NIR & IRTF & 4 \\
\hline 10 & 24-Jun.-2011 & $13: 29$ & 35.4 & 24.8 & 54.6 & 94.0 & $\mathrm{Sr} / \mathrm{Sv}(\mathrm{Sr})$ & NIR & IRTF & 4 \\
\hline 11 & 25-Jul.-2011 & 02:00 & 26.9 & 31.3 & 63.1 & 337.9 & $\mathrm{~S} / \mathrm{Sv}$ & VIS & NTTb & \\
\hline 12 & 25-Jul.-2011 & 02:06 & 26.9 & 31.3 & 63.1 & 324.5 & $\mathrm{~Sv}$ & VIS & NTTb & \\
\hline 13 & 25-Jul.-2011 & $02: 10$ & 26.9 & 31.3 & 63.1 & 315.5 & $\mathrm{~Sv}$ & VIS & $\mathrm{NTTb}$ & \\
\hline 14 & 14-Aug.-2011 & $06: 59$ & 26.9 & 38.5 & 63.1 & 239.0 & $\mathrm{Sr} / \mathrm{Sv}(\mathrm{Sr})$ & NIR & IRTF & 4 \\
\hline 15 & 04-Dec.-2011 & $00: 41$ & 116.3 & 64.0 & -26.3 & 217.8 & $\mathrm{~Sv} / \mathrm{S}$ & VIS & $\mathrm{NTTb}$ & \\
\hline 16 & 04-Dec.-2011 & $00: 43$ & 116.3 & 64.0 & -26.3 & 213.3 & $\mathrm{~Sv}$ & VIS & NTTb & \\
\hline 17 & 04-Dec.-2011 & $02: 11$ & 116.4 & 64.0 & -26.4 & 17.0 & $\mathrm{~S} / \mathrm{Sr} / \mathrm{Sv}$ & VIS & NTTb & \\
\hline 18 & 04-Dec.-2011 & $02: 13$ & 116.4 & 64.0 & -26.4 & 12.5 & $\mathrm{~S} / \mathrm{Sv}$ & VIS & NTTb & \\
\hline 19 & 04-Dec.-2011 & 03:01 & 116.4 & 64.0 & -26.4 & 265.4 & $\mathrm{~S} / \mathrm{Sr} / \mathrm{Sv}$ & VIS & NTTb & \\
\hline 20 & 04-Dec.-2011 & 03:03 & 116.4 & 64.0 & -26.4 & 260.9 & $\mathrm{~S} / \mathrm{Sr} / \mathrm{Sq}$ & VIS & $\mathrm{NTTb}$ & \\
\hline 21 & 02-Feb.-2012 & & 152.4 & 46.0 & -62.4 & & $\mathrm{Sq} / \mathrm{Sr} / \mathrm{S}$ & VIS & PAL & \\
\hline
\end{tabular}

Notes. ID: spectrum identification. Date: listed observing date. UT: observation time when available. Aspect: observing aspect for the nominal pole. $\alpha$ : phase angle. Lat. \& Lon.: asteroid-centric coordinates of the sub-observer point. Spec. class: lists the spectral class as determined here, with literature classification in parentheses (some may be based on earlier taxonomies). Wavelength range: type of spectrum with "VIS" for optical and "NIR" for near infrared, "Facility": code for the instrument used. Ref.: reference to source if the data has been previously published. Facility codes (with MPC codes for the observing site): MDM: MDM observatory McGraw-Hill 2.4-m telescope with Mark III spectrograph (697). IRTF: NASA Infrared Telescope Facility (IRTF) on Mauna Kea with SpeX, US (568). NTTa: ESO NTT telescope with EMMI (809). NTTb: ESO NTT telescope with EFOSC2 (809). PAL: Palomar 200-inch telescope with the Double Spectrograph (675). ${ }^{(a)}$ Unpublished data available at http://smass.mit.edu/data/spex/sp72/, spectral classification provided by Thomas et al. (2014).

References. (1) Binzel et al. (2004); (2) Michelsen et al. (2006); (3) Popescu et al. (2011); (4) Thomas et al. (2014).

the albedo to be determined when using a suitable thermal model. NEATM produces diameters and albedos that are generally accurate to $\sim 15$ and $\sim 30 \%$, respectively (Wolters \& Green 2009). An asteroid's effective diameter (i.e. the equivalent diameter of sphere with the same projected area as the irregularly shaped asteroid), $D_{\text {eff }}$, is related to its absolute visual magnitude, $H$, and geometric albedo, $p_{\mathrm{V}}$, by Fowler \& Chillemi (1992):

$D_{\text {eff }}=\frac{10^{-H / 5} 1329}{\sqrt{p_{\mathrm{V}}}}(\mathrm{km})$.

The Bond albedo, $A_{\mathrm{B}}$, is related to the geometric albedo by

$A_{\mathrm{B}}=(0.290+0.684 G) p_{\mathrm{V}}$,

where $G$ is the phase slope parameter. In the NEATM, the subsolar temperature, $T_{\mathrm{SS}}$, is given by

$T_{\mathrm{SS}}=\left(\frac{\left(1-A_{\mathrm{B}}\right) S}{\eta \varepsilon \sigma}\right)^{\frac{1}{4}}$,

where $S$ is the incident solar flux, $\eta$ is the beaming parameter, $\varepsilon$ is the emissivity ( 0.9 is assumed), and $\sigma$ is the StefanBoltzmann constant. NEATM models the asteroid as a sphere with the day-side temperatures and thermal fluxes calculated assuming Lambertian emission, and with zero temperature or flux assumed on the night-side. The NEATM allows $p_{\mathrm{V}}$ (and, therefore, also $D_{\text {eff }}$ through Eq. (2)) and $\eta$ to be varied to find the best model fit to the observations. The beaming parameter, $\eta$, effectively forces the model to show a colour temperature that is consistent with the apparent colour temperature displayed by the data, and therefore approximately accounts for the effects of thermal inertia and surface roughness on the asteroid thermal emission.

The NEATM was fitted to each VISIR data set independently because it was not possible to fit all three data sets simultaneously due to significant changes in illumination and observation geometry that occurred between the September and December 2011 observations. To determine statistical uncertainties of the fitted NEATM parameters, a bootstrap analysis was performed on the VISIR data where the NEATM was fitted to one hundred synthetic data sets produced by random re-sampling. In each bootstrap trial, the VLT data, and also Cuyo's $H$ and $G$ values, were randomly selected from Gaussian distributions centred on the measured values with FWHMs equal to the measurement uncertainties. The results from each bootstrap trial were then averaged to give the mean values and $1 \sigma$ uncertainties of the fitted NEATM parameters. For this analysis we assumed $H=14.7 \pm 0.3$ and $G=$ $0.23 \pm 0.10$, and Table 5 summarises the results for the three different VISIR data sets. As shown, the NEATM determined an effective diameter of $3.8-4.2 \mathrm{~km}$, a geometric albedo of 0.13-0.16, and a beaming parameter of 1.0-1.3 for Cuyo from the VISIR observations. The rather low beaming parameters 

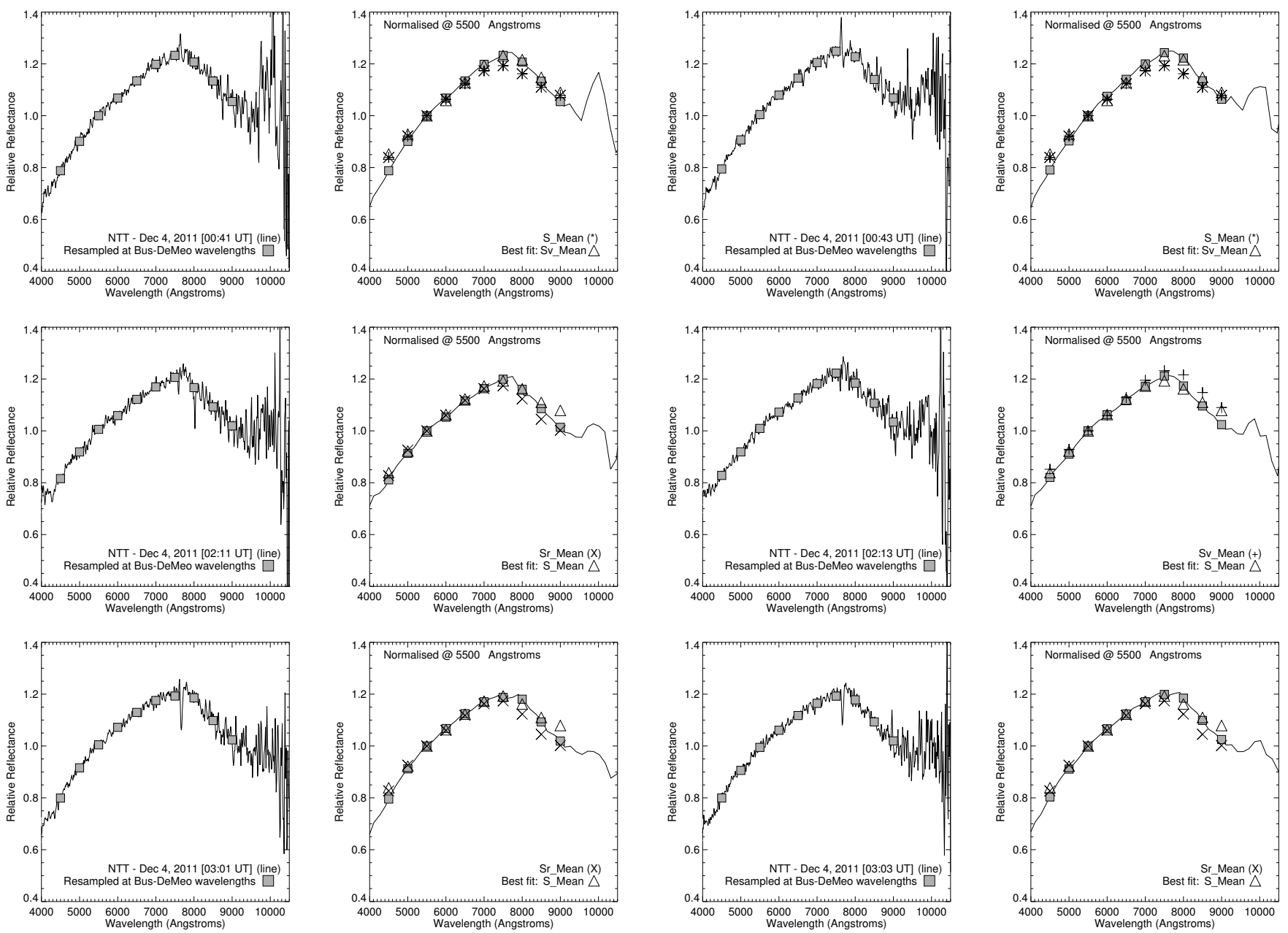

Fig. 8. Similar to Fig. 7, but the spectra were collected at the NTT on December 4, 2011 (also see Fig. 12 and Table 4 (IDs 15-20)).

obtained for moderately high phase angles (i.e. $\sim 45^{\circ}-60^{\circ}$ ) indicates that Cuyo potentially has a low thermal inertia surface. For instance, it has been previously demonstrated that NEATM fits to IR observations of NEAs show a general trend of increasing beaming parameter with increasing phase angle (Wolters \& Green 2009), and a typical beaming parameter value for this phase angle range would be 1.5-1.7. Since the Cuyo beaming parameters are lower than this expected range then this implies that Cuyo has a thermal inertia value that is somewhat lower than the average NEA value of $\sim 200 \mathrm{~J} \mathrm{~m}^{-2} \mathrm{~K}^{-1} \mathrm{~s}^{-1 / 2}$ (Delbo et al. 2007).

\subsection{Results for the Advanced Thermo-Physical Model (ATPM)}

The ATPM was developed to interpret thermal-infrared observations of atmosphere-less planetary surfaces (Rozitis \& Green 2011), and to make more realistic asteroidal Yarkovsky and YORP effect predictions (Rozitis \& Green 2012, 2013). It has previously been applied to several near-Earth asteroids to derive their thermo-physical and dynamical properties (e.g. Lowry et al. 2014; Rozitis 2017; Rozitis \& Green 2014; Rozitis et al. 2013, 2014, 2018; Wolters et al. 2011). To briefly summarise how it works, the ATPM computes the surface temperature distribution of an irregularly shaped asteroid by solving the 1D heat conduction equation for each facet of the asteroid's shape model.
To ensure energy balance between incoming (i.e. direct sunlight) and outgoing (i.e. thermal-infrared emission) radiation, a surface boundary condition is also solved for each facet. Rough surface thermal-infrared beaming (i.e. re-radiation of absorbed sunlight back towards the Sun) is taken into account by including a fractional coverage, $f_{\mathrm{R}}$, of hemispherical craters on each shape model facet. The hemispherical crater (i.e. with crater opening angle of $180^{\circ}$ ) has previously been demonstrated to accurately reproduce the beaming effects observed for the Moon (Rozitis \& Green 2011) and the near-Earth asteroid (433) Eros (Rozitis 2017). The ATPM is run for a range of thermo-physical properties, and the model thermal emission is computed from the sum of the Planck function for all facets that were visible to the observer at the time of the observations.

The Cuyo shape model was provided by the light curve inversion described earlier, and the free parameters to be constrained in ATPM fits to the thermal-infrared observations were the effective diameter, $D_{\text {eff }}$, thermal inertia, $\Gamma$, and surface roughness fraction, $f_{\mathrm{R}}$. The light curve rotational phasing information had sufficient accuracy to allow computation of the exact rotation phase of Cuyo for each thermal-infrared flux measurement, and so it was not necessary to have rotation phase as an additional free parameter. The model fluxes, $F_{\mathrm{MOD}}\left(\lambda_{n}, D, \Gamma, f_{\mathrm{R}}\right)$, were compared with the observations, $F_{\mathrm{OBS}}\left(\lambda_{n}\right)$, and observational errors, $\sigma_{\mathrm{OBS}}\left(\lambda_{n}\right)$, by varying the three free parameters to give the 

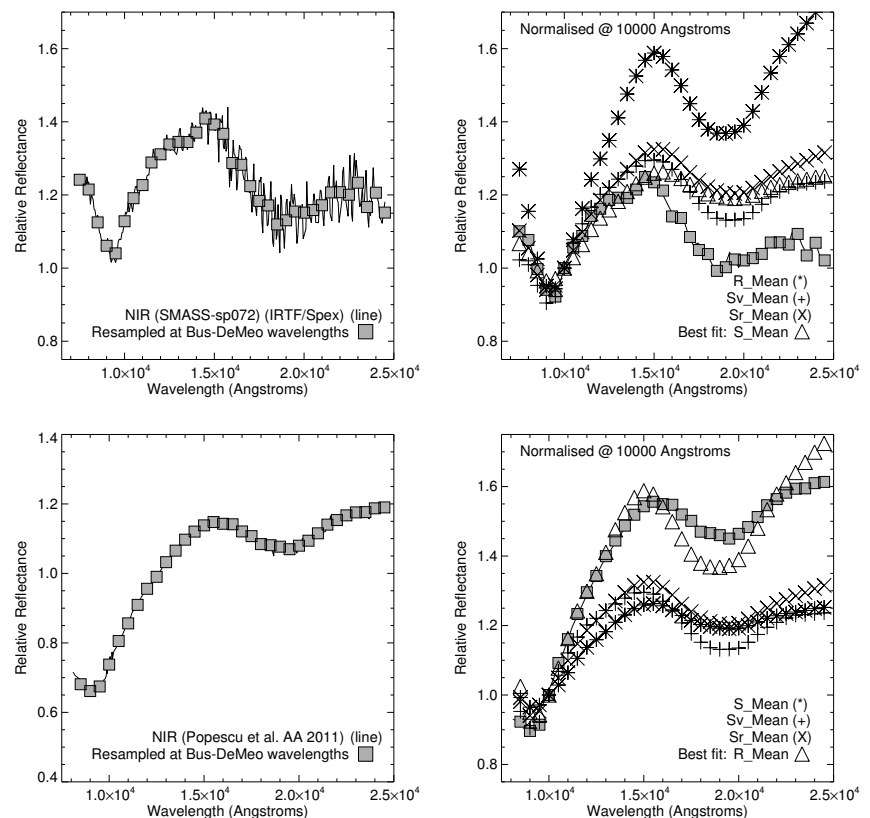

Fig. 9. Near-IR spectra obtained using the NASA IRTF telescope, on the nights of July 7, 2008 (top panels) (SMASS: see Table 4 for reference) and August 27, 2008 (bottom panels) (Popescu et al. 2011). The panels on the left are normalised at the wavelengths used in the original publications: $5500 \AA$ for the SMASS spectrum and $12500 \AA$ for the Popescu et al. spectrum. The plots on the right have been re-normalised at $10000 \AA$ for consistency and easier comparison with spectra from Fig. 10. These spectra correspond to IDs " 3 " and "4" in Table 4. Each spectrum was centred at near $0^{\circ}$ latitude, but at near opposite ends of the asteroid in terms of longitude or rotation phase (also see Fig. 12). The spectra have very different properties, perhaps indicating compositional differences across the asteroid near the equatorial region. This observation holds regardless of where the spectra are normalised.

$\operatorname{minimum} \chi^{2}$ fit,

$\chi^{2}=\sum_{n=1}^{N}\left(\frac{\mathrm{FCF}(D) F_{\mathrm{MOD}}\left(\lambda_{n}, D, \Gamma, f_{\mathrm{R}}\right)-F_{\mathrm{OBS}}\left(\lambda_{n}\right)}{\sigma_{\mathrm{OBS}}\left(\lambda_{n}\right)}\right)^{2}$,

for a set of $n=1$ to $N$ observations with wavelength $\lambda_{n}$. A flux correction factor FCF $(D)$ was used that took into account adjustments to the Bond albedo when a fixed value was used in the temperature modelling (i.e. different diameters require different Bond albedos as dictated by Eqs. (1) and (2)), and saves computational effort by not having to run the ATPM for multiple values (see Wolters et al. 2011; Rozitis et al. 2013, for more details of this methodology in use). A model Bond albedo value of 0.15 was used in the ATPM, which gave flux correction factors that were within $10 \%$ of unity. For each observational data set, the ATPM was run for the nominal Cuyo shape model using the geometry given in Table 2 . The thermal inertia was initially varied between 0 and $500 \mathrm{~J} \mathrm{~m}^{-2} \mathrm{~K}^{-1} \mathrm{~s}^{-1 / 2}$ in equally spaced steps of $10 \mathrm{~J} \mathrm{~m}^{-2} \mathrm{~K}^{-1} \mathrm{~s}^{-1 / 2}$, and then between 0 and $100 \mathrm{~J} \mathrm{~m}^{-2} \mathrm{~K}^{-1} \mathrm{~s}^{-1 / 2}$ in equally spaced steps of $2 \mathrm{~J} \mathrm{~m}^{-2} \mathrm{~K}^{-1} \mathrm{~s}^{-1 / 2}$ when the likely thermal inertia range had initially been constrained. The effective diameter and roughness fraction were also stepped through their plausible ranges to form a 3D grid of model test parameters (or clones). The minimum $\chi^{2}$ fit was sought for within the 3D grid, and a region bounded by a constant $\Delta \chi^{2}$ value at the $3 \sigma$ confidence level (i.e. $\Delta \chi^{2}=14.2$ for three free parameters) then defined the range of possible parameters or clones around the best fit. As in Wolters et al. (2011) and Rozitis et al. (2018), all acceptable clones were averaged to give a mean and standard deviation of the fitted parameters. The uncertainties derived by this method were checked for realism by applying an alternative bootstrap fitting technique to the VISIR data (i.e. see Rozitis et al. 2018, for the bootstrap methodology used). Finally, a $2.5 \%$ uncertainty was added in quadrature to the statistical diameter uncertainty to take into account absolute calibration uncertainties of the VISIR data. This is because ground-based thermal-infrared observations have an estimated absolute calibration uncertainty that ranges from 7 to $10 \%$ (Lim et al. 2005; Wolters et al. 2008). However, since the VISIR observations were calibrated on three separate nights then the combined absolute calibration uncertainty is reduced to $5 \%$, which translates to an additional $2.5 \%$ uncertainty on the derived diameter.

The ATPM was fitted to all three VISIR data sets of Cuyo simultaneously. For the nominal shape model, the ATPM fit gave an effective diameter of $3.13 \pm 0.08 \mathrm{~km}$, a thermal inertia of $45 \pm 8 \mathrm{~J} \mathrm{~m}^{-2} \mathrm{~K}^{-1} \mathrm{~s}^{-1 / 2}$, and a roughness fraction of $0.56 \pm 0.24$ with a model fit reduced- $\chi^{2}$ of 3.93 . This reduced- $\chi^{2}$ value was rather large and indicated that the ATPM was not reproducing some aspect of the data. As shown in Fig. 13, the ATPM reproduced the thermal light curves of Cuyo rather well except for two deep and very narrow dips seen in the data obtained on the nights of September 5 and December 17. These features were likely caused by short changes in weather conditions at the VLT during the observations. The dips were too deep for an eclipse/occultation event caused by a hypothetical undiscovered moon of Cuyo, and the radar observations by Ostro \& Wisniewski (1992) did not detect the presence of any relatively large moons in orbit around Cuyo. The dips were also too narrow for a hypothetical spot on the surface that had different thermal properties to the rest of Cuyo's surface, as hemispherical averaging would have made detections of this very difficult. Ignoring the data contained in the dips, the ATPM fit produced a more reasonable reduced- $\chi^{2}$ value of 1.52 , and the effective diameter, thermal inertia, and roughness fraction were derived to be $3.15 \pm 0.08 \mathrm{~km}, 44 \pm 9 \mathrm{~J} \mathrm{~m}^{-2} \mathrm{~K}^{-1} \mathrm{~s}^{-1 / 2}$, and $0.52 \pm 0.26$, respectively. These results are summarised in Table 6 , and the $\Delta \chi^{2}$ fitting contours are shown in Fig. 14. The very low thermal inertia value derived here is consistent with the rather low beaming parameters determined by NEATM, and is similar to the very low thermal inertia value of $24_{-14}^{+20} \mathrm{~J} \mathrm{~m}^{-2} \mathrm{~K}^{-1} \mathrm{~s}^{-1 / 2}$ determined previously for the near-Earth asteroid (29075) 1950 DA (Rozitis et al. 2014). The diameter derived by the ATPM is somewhat smaller than that derived by the NEATM but this is not unusual because the NEATM assumes a spherical shape and has a tendency to overestimate asteroid diameters when asteroids are observed at large phase angle (Wolters \& Green 2009).

The Cuyo shape model and its thermo-physical analysis were checked for accuracy by comparing the effective diameters derived by the ATPM with diameters measured by Doppler-radar observations taken in September 1989 (Ostro \& Wisniewski 1992). In particular, they determined that the maximum equatorial diameter of Cuyo was $3.9 / \cos (\delta) \mathrm{km}$, where $\delta$ was the sub-radar latitude on Cuyo at the time of their observations. Using the shape model pole orientation found earlier, the subradar latitude was determined to be $29.0^{\circ}$ for the nominal shape model of Cuyo. This sub-radar latitude then gave the maximum equatorial diameter of Cuyo to be $4.46 \pm 0.45 \mathrm{~km}$ (i.e. a $10 \%$ uncertainty is typically applied to radar-derived diameters). Taking into account the irregular shape of Cuyo, this maximum equatorial diameter corresponded to an effective diameter of $3.45 \pm 0.35 \mathrm{~km}$ for the nominal shape model. This compares well 

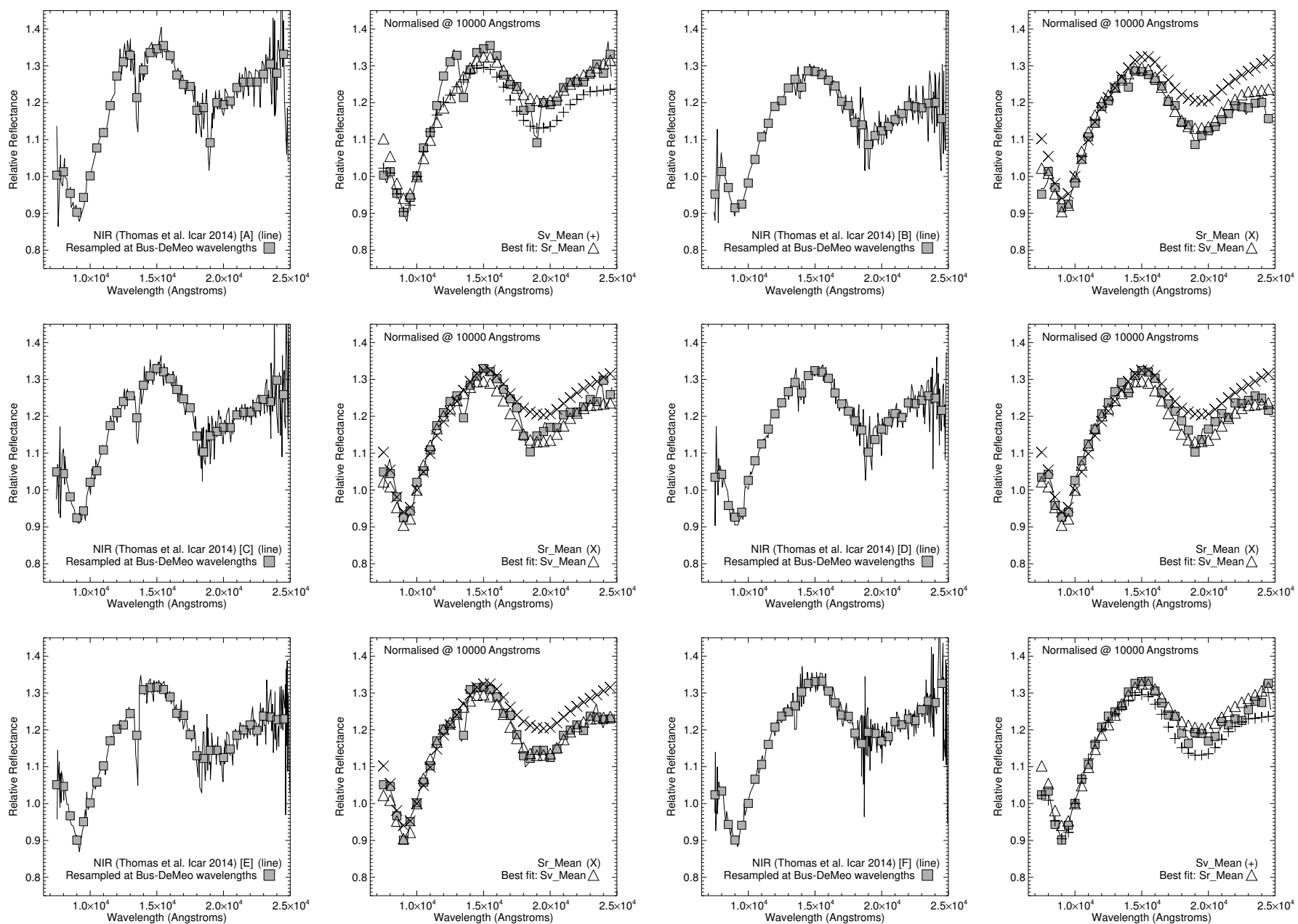

Fig. 10. Near-IR spectra obtained using the NASA IRTF telescope, on the night of June 24, 2011 (Thomas et al. 2014). The spectra cover just over one full rotation of the asteroid. Linking the timings of the spectra to our spin-state solution may indicate potential compositional variation at high latitudes. The spectra are labelled as "A"-"F" in line with the original labelling used by Thomas et al. (these correspond to IDs 5-10 in Table 4). The spectra are normalised at $10000 \AA$.
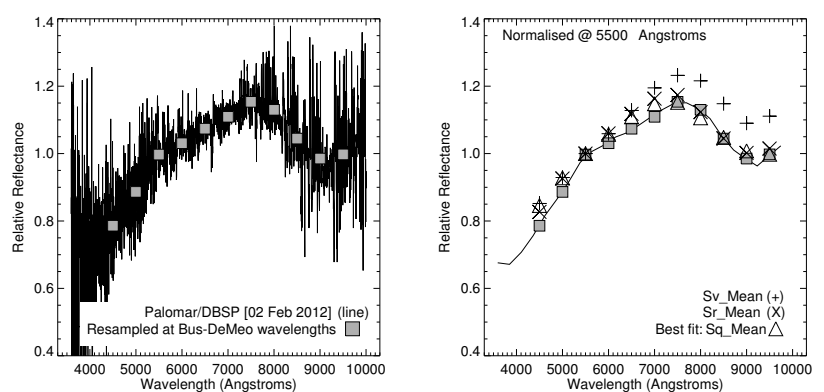

Fig. 11. Spectrum of (1917) Cuyo taken at Palomar Observatory on February 2, 2012. Here the spectrum is most consistent with the Sq type (also see Fig. 12 and Table 4 (ID 21)).

to the effective diameter derived by the ATPM described earlier, and indicates that the light curve shape model of Cuyo is accurate. Typically, an inaccurate shape model would give inconsistent measurements of diameter by independent thermal-infrared and radar observations (e.g. Rozitis et al. 2013; Rozitis \& Green 2014).

For comparisons with the light curve YORP constraints, the YORP effect acting on Cuyo could be predicted by computing the total recoil forces and torques from reflected and thermally emitted photons from the asteroid surface using the ATPM. These calculations were made for both a smooth and rough surface, and were averaged over both the asteroid rotation and elliptical orbit (see Rozitis \& Green 2012, 2013, for methodology). As demonstrated in Rozitis \& Green (2012), the inclusion of rough-surface thermal-infrared beaming effects in the YORP predictions tends to dampen the YORP rotational acceleration on average but can add uncertainties of up to several tens of per cent if the roughness was varied across the surface. Since the light curve inversion produced convex shape models only, then shadowing and self-heating effects inside global-scale concavities (see Rozitis \& Green 2013) were not possible to model. However, a study of non-convex shape models for fast two to four hour rotators in Rozitis \& Green (2013) indicated that such asteroids have rather minimal levels of global-scale concavities, and the $\sim 2.7 \mathrm{~h}$ rotation period of Cuyo implies that its shape could be similar. Furthermore, the Tangential-YORP effect, that is, a predicted rotational acceleration caused by temperature asymmetries within exposed rocks and boulders on the surface of an asteroid (Golubov \& Krugly 2012), was also not included in the ATPM predictions. However, the very low thermal inertia value measured for Cuyo implies the absence of rocks and boulders on its surface of the quantity and size that are necessary to induce a significant Tangential-YORP component. As 


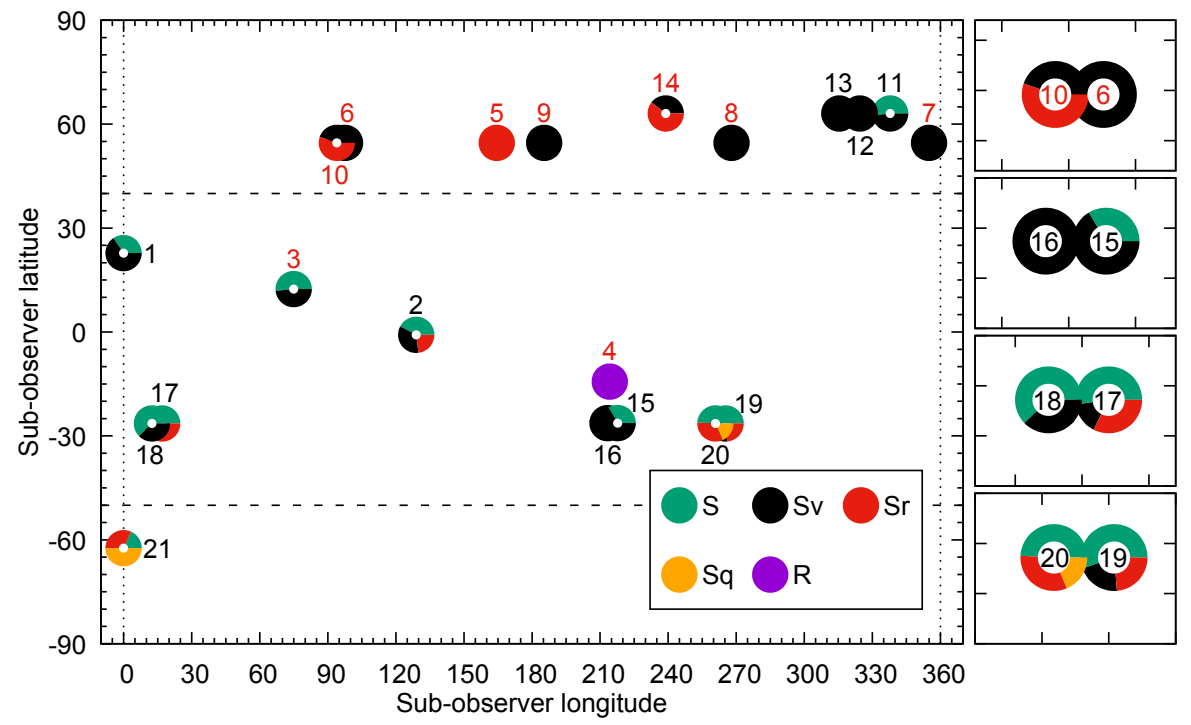

Fig. 12. Geometry at which each spectrum of Cuyo was taken. For each spectrum the location of the symbol is determined by sub-observer latitude and longitude in asteroid-centric coordinates. The spectra are labelled by their ID, as listed in Table 4. Spectra taken at near infrared wavelengths are labelled with red numbers, and at the optical wavelengths in black. The symbols are colour-coded and shown in the legend. Closed symbols are used for spectra where there was a very definitive match to one of the spectral classes, while open multi-coloured symbols are used to demonstrate the relative ambiguity in spectral class determination. On the right, we replot those cases where data points overlap, for clarity.
Table 5. Results of the NEATM thermal analysis of (1917) Cuyo using the ESO VLT VISIR data.

\begin{tabular}{ccccccc}
\hline \hline Date & $D_{\text {eff }}(\mathrm{km})$ & $\sigma$ & $p_{\mathrm{V}}$ & $\sigma$ & $\eta$ & $\sigma$ \\
\hline $04 / 09 / 11$ & 3.81 & ${ }_{-0.12}^{+0.12}$ & 0.16 & ${ }_{-0.03}^{+0.05}$ & 1.27 & ${ }_{-0.08}^{+0.08}$ \\
& & & & \\
$16 / 12 / 11$ & 4.18 & ${ }_{-0.14}^{+0.16}$ & 0.13 & ${ }_{-0.03}^{+0.05}$ & 1.25 & ${ }_{-0.09}^{+0.10}$ \\
& & & & & \\
$18 / 12 / 11$ & \multirow{2}{*}{3.80} & ${ }_{-0.37}^{+0.35}$ & 0.16 & ${ }_{-0.04}^{+0.07}$ & 0.97 & ${ }_{-0.21}^{+0.20}$ \\
\hline
\end{tabular}

Cuyo is likely to be an S-type rubble-pile asteroid, a bulk density equivalent to that measured for the S-type rubble-pile asteroid (25143) Itokawa (Abe et al. 2006) of $2 \mathrm{~g} \mathrm{~cm}^{-3}$ was assumed for the YORP computations. Using the thermo-physical properties derived earlier, the ATPM predicts YORP rotational acceleration of $(-6.39 \pm 0.96) \times 10^{-10} \mathrm{rad} \mathrm{day}^{-2}$ for the nominal shape model. The uncertainty given here corresponds to the standard deviation of results when the degree of surface roughness is randomly varied across the surface of Cuyo (see Lowry et al. 2014, for details of the Monte Carlo methodology used). These values lie well within the light curve rotational acceleration constraints determined previously.

\section{Summary and main conclusions}

The analysis of light curve data for Cuyo allowed a determination of a robust convex shape model using recent observations and archived data from 1989 and 1995. Currently, a YORP-induced rotation-rate change over the entire time frame of our data cannot be confirmed, although the range of possible $v$ values was constrained.

One possible source of a large uncertainty in the spin-up measurements can be linked to the quality of the archive data sets. Given the very low YORP strength that this asteroid seems to have, the observations can effectively be divided into two epochs: the earliest observations from 1989 together with the ESO Danish telescope light curves from 1995, and the most recent observations from 2008-2013. In terms of looking for a quadratic trend in rotational phase offsets, as was found for Itokawa (Lowry et al. 2014, Fig. 1), the YORP fitting includes deciding whether a parabola or a straight line fits two points better. To measure any possible YORP value an additional data point would be required to determine the quadratic trend in the phase offset change with time, or an independent shape and pole orientation estimate, perhaps by radar during the next radar opportunity in 2032 .

Cuyo has a diamond-like shape, characteristic of a rapidly rotating rubble pile (Sánchez \& Scheeres 2016), which suggests that it is an evolved system. The lack of measurable spin-up by the YORP effect for this object might be a result of the YORP effect's self-limiting properties (Cotto-Figueroa et al. 2015). The reorientation of surface material can reduce the body asymmetry leading to a decrease of the YORP magnitude.

We used our robust shape and spin-state model to investigate spectroscopic properties of the asteroid. The shape model allowed us to determine the precise observational geometry for our new spectra and all spectra available in the literature, taken within the time span of the photometry used in the model solution. These data include spectra taken at optical and near-IR wavelengths. We find that there may be tentative evidence of compositional differences between the southern pole and the rest of the body, based on the optical spectra alone. However, much stronger differences can be seen in the near-IR spectra, indicative of varying surface composition along the equatorial region. More specifically, there appears to be significant compositional differences at near-opposite sides of the body, where the near-IR spectra vary from $S$ type to $\mathrm{R}$ type, in the BusDeMeo taxonomy (Bus \& Binzel 2002; DeMeo et al. 2009). We stress though that we are more concerned here with the perceived relative differences in the spectra across these two regions of the asteroid, rather than the actual taxonomic classification or surface composition. The observational geometry was very similar when the spectra were taken by the different observers, in terms of aspect and phase angles. Furthermore, there may also be evidence of a patch of less-weathered material around longitude $165^{\circ}$, but at high latitudes. This is based on a set of near-IR spectra taken across a single full rotation of the body and presumably processed using identical procedures at each rotation phase (Thomas et al. 2014). So in this case, differences between observational geometry and data-reduction process cannot account for the differences seen in the spectra (although the differences between the near-IR spectra at equatorial latitudes is significant enough to preclude this possibility here too). 

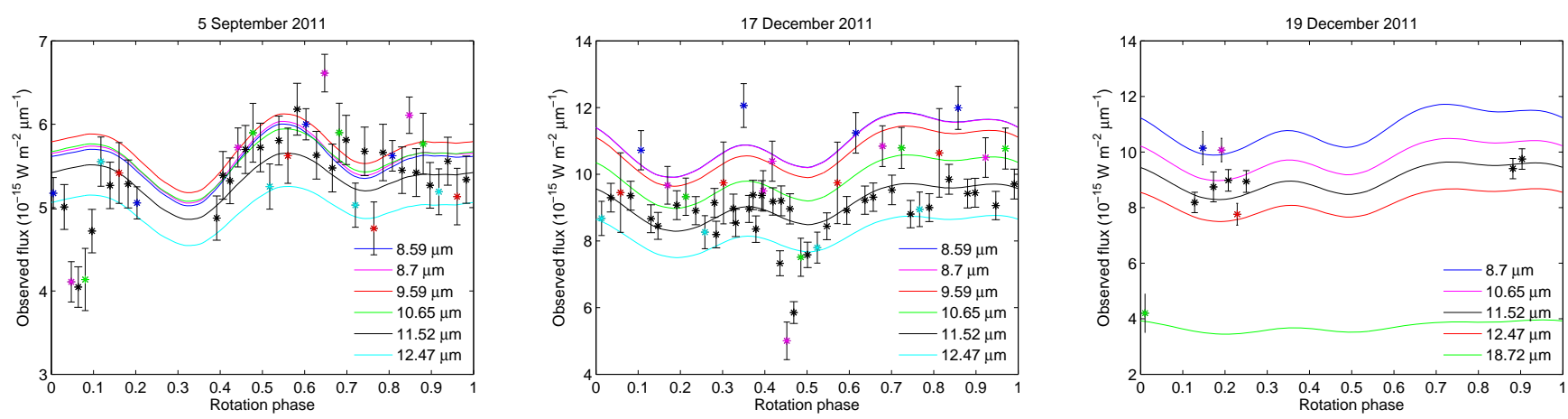

Fig. 13. Thermal-infrared fits of the ATPM to the ESO VLT VISIR data of (1917) Cuyo. This fit was obtained using the nominal shape model with a thermal inertia of $44 \mathrm{~J} \mathrm{~m}^{-2} \mathrm{~K}^{-1} \mathrm{~s}^{-1 / 2}$ and a surface roughness fraction of 0.5 . The dips in the observed flux of Cuyo at rotation phases of $\sim 0.05$ and $\sim 0.45$ on September 5 and December 17, respectively, are likely due to short changes in weather conditions and, therefore, were not considered further in our analysis. The error bars represent the $1 \sigma$ uncertainties on the measured flux data points, and the different wavelengths used are colour-coded.

Table 6. Results of the ATPM thermo-physical analysis of (1917) Cuyo using the ESO VLT VISIR data.

\begin{tabular}{ll}
\hline \hline$D_{\text {eff }}(\mathrm{km})$ & $3.15 \pm 0.08$ \\
$p_{\mathrm{V}}$ & $0.24 \pm 0.07$ \\
$\Gamma\left(\mathrm{J} \mathrm{m}^{-2} \mathrm{~K}^{-1} \mathrm{~s}^{-1 / 2}\right)$ & $44 \pm 9$ \\
$f_{\mathrm{R}}$ & $0.52 \pm 0.26$ \\
Reduced $\chi^{2}$ & 1.52 \\
$v\left(\times 10^{-10} \mathrm{rad} \mathrm{day}^{-2}\right)$ & $-6.39 \pm 0.96$ \\
$D_{\text {radar }}(\mathrm{km})$ & $3.45 \pm 0.35$ \\
\hline
\end{tabular}

Notes. The table lists: the effective diameter $\left(D_{\text {eff }}\right)$, geometric albedo $\left(p_{\mathrm{V}}\right)$, thermal inertia $(\Gamma)$, surface roughness fraction $\left(f_{\mathrm{R}}\right)$, reduced $\chi^{2}$, predicted YORP rotational acceleration $(v)$, and the radar-derived diameter $\left(D_{\text {radar }}\right)$.

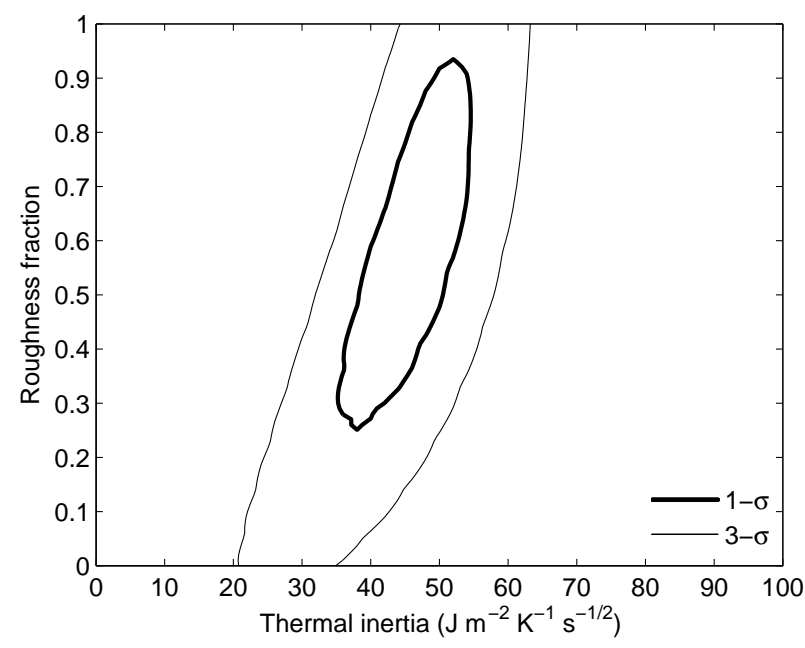

Fig. 14. Results of $\chi^{2}$ fitting the ATPM to the ESO VLT VISIR data of (1917) Cuyo. The thick and thin contours indicate the delta-chi-squared cut-off boundaries for $1 \sigma$ and $3 \sigma$ confidence levels, respectively.

This finding is important given the current shape and spin state that we determine. The equatorial bulge implies a deep rubble-pile sub-surface structure and therefore the movement of material on the surface is highly likely when the body is subjected to thermal torques. The varying spectral signatures across the surface may be the result of such movement, which would also be consistent with the spin rate of the asteroid, currently at its spin-fission limit. At the time of writing, new images were released of the "spinning-top" or "YORPoid"-shaped asteroid Ryugu from the Hayabusa 2 mission (Watanabe et al. 2017). These spectacular images show a body highly evolved due to YORP, and possibly catastrophic collision(s). There may be opportunity to search for spectral evidence of regolith movement, although the asteroid is no longer spinning near its fission limit. It is therefore likely to have a more homogeneous surface compositionally, having been exposed to space weathering for, presumably, a long period.

The high spin rate makes Cuyo a target of interest for the study of cohesive forces preventing asteroids from breaking up (Rozitis et al. 2014). Cuyo has very similar surface thermal inertia to the asteroid (29075) 1950 DA on which the effect was studied before, however, it is larger and rotates more slowly. As Cuyo is still close to the spin-fission limit, there is a question of whether the cohesive forces are enough to keep the fine-grain material on the surface. This issue is being further investigated and will be discussed in an upcoming paper.

Acknowledgements. A.R., S.C.L., S.F.G., C.S., and A.F. acknowledge support from the UK Science and Technology Facilities Council. A.R. and S.C.L. acknowledge support from the Southeast Physics Network (SEPnet). B.R. acknowledges support from the Royal Astronomical Society (RAS) in the form of a research fellowship. We thank all the staff at the observatories involved in this study for their support. This work is based on observations collected at the European Organisation for Astronomical Research in the Southern Hemisphere under ESO programmes 185.C-1033 and 185.C-1034. The portion of this research by K.J.L. was carried out at the Jet Propulsion Laboratory, California Institute of Technology, under contract with the National Aeronautics and Space Administration. This work was based in part on observations at the following observatories: Table Mountain Observatory, California; Palomar Observatory, California. We would like to thank T. Michałowski and A. Erikson, for providing the archived light curve data. Some of the archive data were available from the (now decommissioned) Asteroid Photometric Catalogue (Piironen et al. 2001). We would like to thank $\mathrm{C}$. Thomas for providing the archived spectroscopic data. The Image Reduction and Analysis Facility (IRAF) software was used for data reduction. IRAF is distributed by the National Optical Astronomy Observatory, which is operated by the Association of Universities for Research in Astronomy (AURA) under a cooperative agreement with the National Science Foundation. The asteroid ephemeris were generated using JPL's Horizons service (Giorgini et al. 1996). We utilized data from the SMASS archive in this study (Rayner et al. 2003).

\section{References}

Abe, M., Takagi, Y., Kitazato, K., et al. 2006, Science, 312, 1334 Binzel, R. P., Rivkin, A. S., Stuart, J. S., et al. 2004, Icarus, 170, 259 


\section{A. Rożek et al.: Physical model of near-Earth asteroid (1917) Cuyo}

Bottke, Jr. W. F., Vokrouhlický, D., Rubincam, D. P., \& Broz, M. 2002, Asteroids III, eds. B. Bottke, C. Cellino, P. Paolicchi, \& R. P. Binzel (Tucson: University of Arizona Press), 395

Bus, S. 1999, Ph.D. Thesis, Massachusetts Institute of Technology, Cambridge, USA

Bus, S. J., \& Binzel, R. P. 2002, Icarus, 158, 146

Buzzoni, B., Delabre, B., Dekker, H., et al. 1984, The Messenger, 38, 9

Chesley, S. R., Ostro, S. J., Vokrouhlický, D., et al. 2003, Science, 302, 1739

Cohen, M., Walker, R. G., Carter, B., et al. 1999, AJ, 117, 1864

Cotto-Figueroa, D., Statler, T. S., Richardson, D. C., \& Tanga, P. 2015, ApJ, 803,

DeMeo, F. E., Binzel, R. P., Slivan, S. M., \& Bus, S. J. 2009, Icarus, 202, 160

Delbo, M., dell'Oro, A., Harris, A., Mottola, S., \& Mueller, M. 2007, Icarus, 190, 236

Duddy, S. R., Lowry, S. C., Wolters, S. D., et al. 2012, A\&A, 539, A36

Durech, J., Vokrouhlický, D., Kaasalainen, M., et al. 2008, A\&A, 489, L25

Durech, J., Sidorin, V., \& Kaasalainen, M. 2010, A\&A, 513, A46

Ďurech, J., Vokrouhlický, D., Baransky, A. R., et al. 2012, A\&A, 547, A10

Durech, J., Vokrouhlický, D., Pravec, P., et al. 2018, A\&A, 609, A86

Erikson, A., Mottola, S., Lagerros, J. S. V., et al. 2000, Icarus, 147, 487

Fowler, J. W., \& Chillemi, J. R. 1992, IRAS asteroid data processing (MA Phillips Laboratory), 17

Giorgini, J. D., Yeomans, D. K., Chamberlin, A. B., et al. 1996, BAAS, 28, 1158

Golubov, O., \& Krugly, Y. N. 2012, ApJ, 752, L11

Golubov, O., Scheeres, D. J., \& Krugly, Y. N. 2014, ApJ, 794, 22

Harris, A. W. 1998, Icarus, 131, 291

Hoffmann, M., Rebhan, H., Neukum, G., \& Geyer, E. H. 1993, Acta Astron., 43, 61

Kaasalainen, M., \& Torppa, J. 2001, Icarus, 153, 24

Kaasalainen, M., Torppa, J., \& Muinonen, K. 2001, Icarus, 153, 37

Kaasalainen, M., Durech, J., Warner, B. D., Krugly, Y. N., \& Gaftonyuk, N. M. 2007, Nature, 446, 420

Lagage, P. O., Pel, J. W., Authier, M., et al. 2004, The Messenger, 117, 12

Lim, L. F., McConnochie, T. H., Bell, J. F., et al. 2005, Icarus, 173, 585

Lowry, S. C., Fitzsimmons, A., Pravec, P., et al. 2007, Science, 316, 272

Lowry, S. C., Weissman, P. R., Duddy, S. R., et al. 2014, A\&A, 562, A48

Michelsen, R., Nathues, A., \& Lagerkvist, C.-I. 2006, A\&A, 451, 331

Oke, J. B., \& Gunn, J. E. 1982, PASP, 94, 586

Ostro, S. J., \& Wisniewski, W. Z. 1992, in Asteroids, Comets, Meteors 1991, eds. A. W. Harris \& E. Bowell (Houston: Lunar and Planetary Institute), 447
Ostro, S. J., Connelly, R., \& Dorogi, M. 1988, Icarus, 75, 30

Ostro, S. J., Margot, J.-L., Benner, L. A. M., et al. 2006, Science, 314, 1276

Pantin, E., Vanzi, L., \& Weilenmann, U. 2008, in 2007 ESO Instrument Calibration Workshop, eds. A. Kaufer \& F. Kerber (Berlin: Springer Science \& Business Media), 119

Piironen, J., Lagerkvist, C.-I., Torppa, J., Kaasalainen, M., \& Warner, B. 2001, BAAS, 33, 1562

Popescu, M., Birlan, M., Binzel, R., et al. 2011, A\&A, 535, A15

Rayner, J. T., Toomey, D. W., Onaka, P. M., et al. 2003, PASP, 115, 362

Rozitis, B. 2017, MNRAS, 464, 915

Rozitis, B., \& Green, S. F. 2011, MNRAS, 415, 2042

Rozitis, B., \& Green, S. F. 2012, MNRAS, 423, 367

Rozitis, B., \& Green, S. F. 2013, MNRAS, 433, 603

Rozitis, B., \& Green, S. F. 2014, A\&A, 568, A43

Rozitis, B., Duddy, S. R., Green, S. F., \& Lowry, S. C. 2013, A\&A, 555, A20

Rozitis, B., Maclennan, E., \& Emery, J. P. 2014, Nature, 512, 174

Rozitis, B., Green, S. F., MacLennan, E., \& Emery, J. P. 2018, MNRAS, 477, 1782

Rubincam, D. P. 2000, Icarus, 148, 2

Scheeres, D. J., Fahnestock, E. G., Ostro, S. J., et al. 2006, Science, 314, 1280

Shoemaker, E. M., \& Helin, E. F. 1978, in NASA Conference Publication, eds. D. Morrison \& W. C. Wells (Washington: NASA, Scientific and Technical Information Office), 2053

Snodgrass, C., \& Carry, B. 2013, The Messenger, 152, 14

Sánchez, P., \& Scheeres, D. J. 2016, Icarus, 271, 453

Taylor, P. A., Margot, J.-L., Vokrouhlický, D., et al. 2007, Science, 316, 274

Tholen, D. J. 1984, PhD Thesis, University of Arizona, Tucson, USA

Thomas, C. A., Emery, J. P., Trilling, D. E., et al. 2014, Icarus, 228, 217

Velichko, F. P., Lupishko, D. F., \& Shevchenko, V. G. 1989, Astron. Tsirk., 1541, 35

Vereš, P., Jedicke, R., Fitzsimmons, A., et al. 2015, Icarus, 261, 34

Walsh, K. J., Richardson, D. C., \& Michel, P. 2008, Nature, 454, 188

Walsh, K. J., Richardson, D. C., \& Michel, P. 2012, Icarus, 220, 514

Watanabe, S.-i., Tsuda, Y., Yoshikawa, M., et al. 2017, Space Sci. Rev., 208, 3

Wisniewski, W. Z., Michałowski, T. M., \& Harris, A. W., \& McMillan, R. S. 1997, Icarus, 126, 395

Wolters, S. D., \& Green, S. F. 2009, MNRAS, 400, 204

Wolters, S. D., Green, S. F., McBride, N., et al. 2008, Icarus, 193, 535

Wolters, S. D., Rozitis, B., Duddy, S. R., et al. 2011, MNRAS, 418, 1246 


\section{Appendix A: Additional figures}
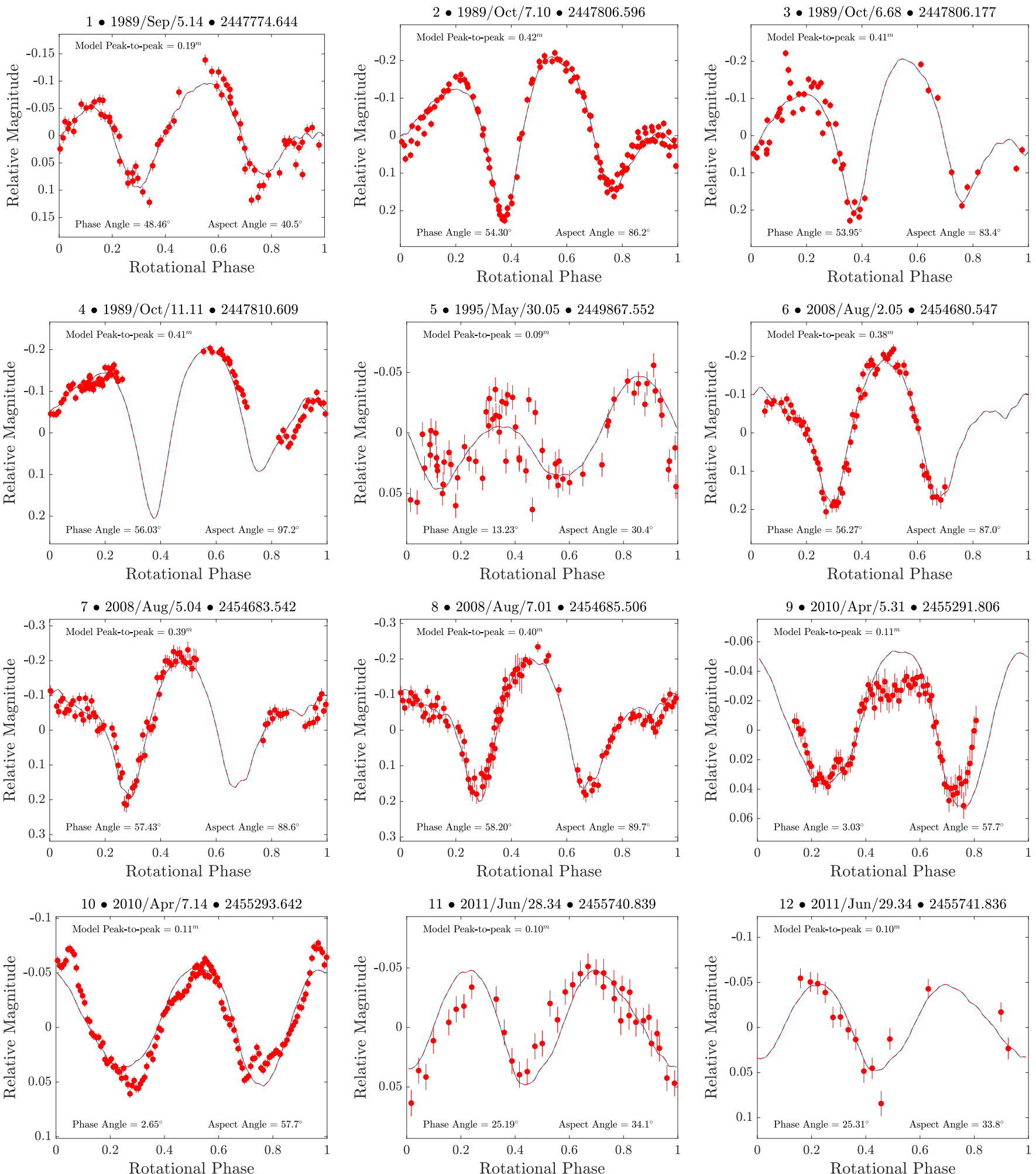

Fig. A.1. Comparison of synthetic light curves generated for Cuyo using the nominal shape model and a constant rotation-period, with the corresponding observed light curves. 
A. Rożek et al.: Physical model of near-Earth asteroid (1917) Cuyo
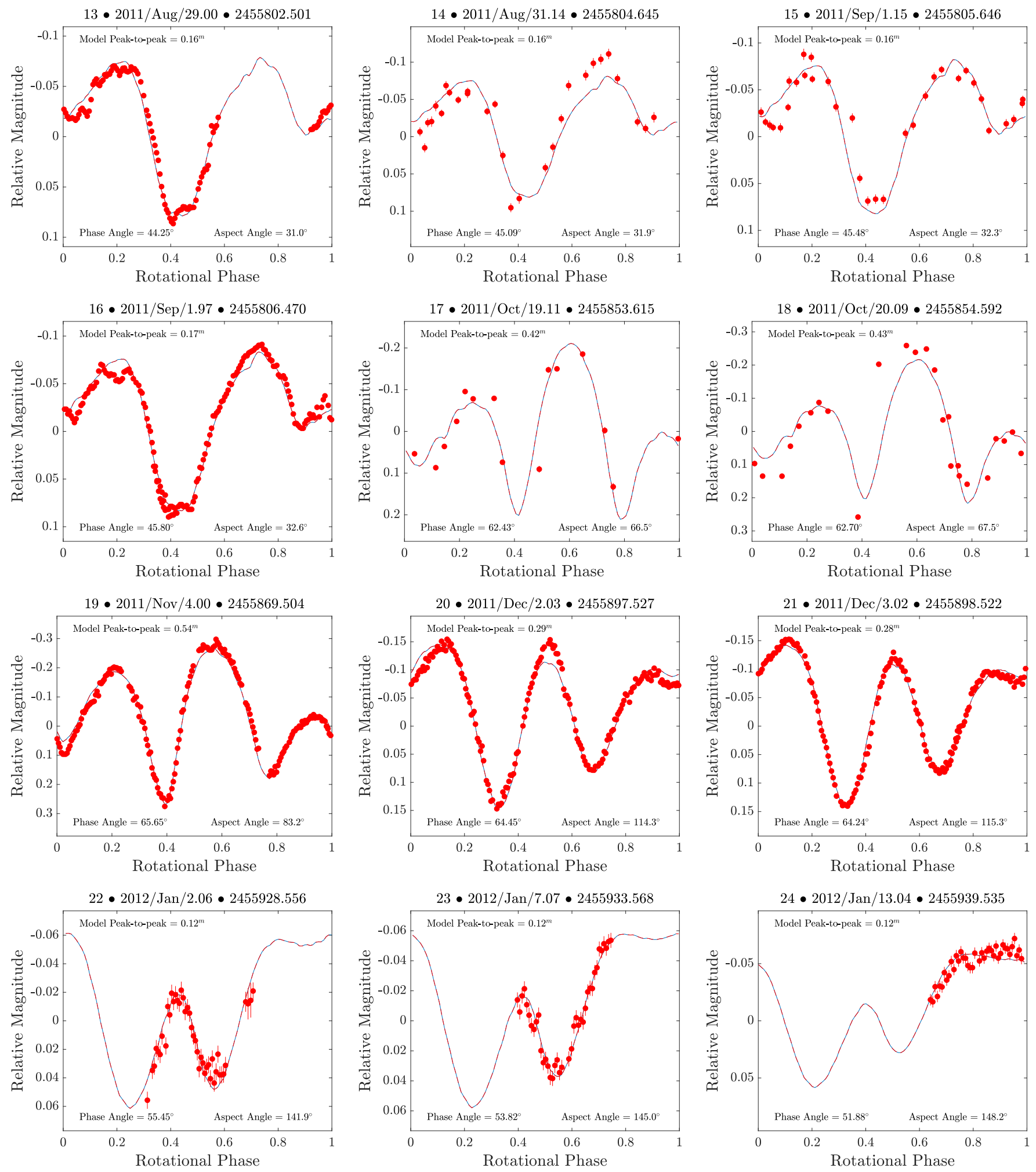

Fig. A.1. continued. 
A\&A 627, A172 (2019)
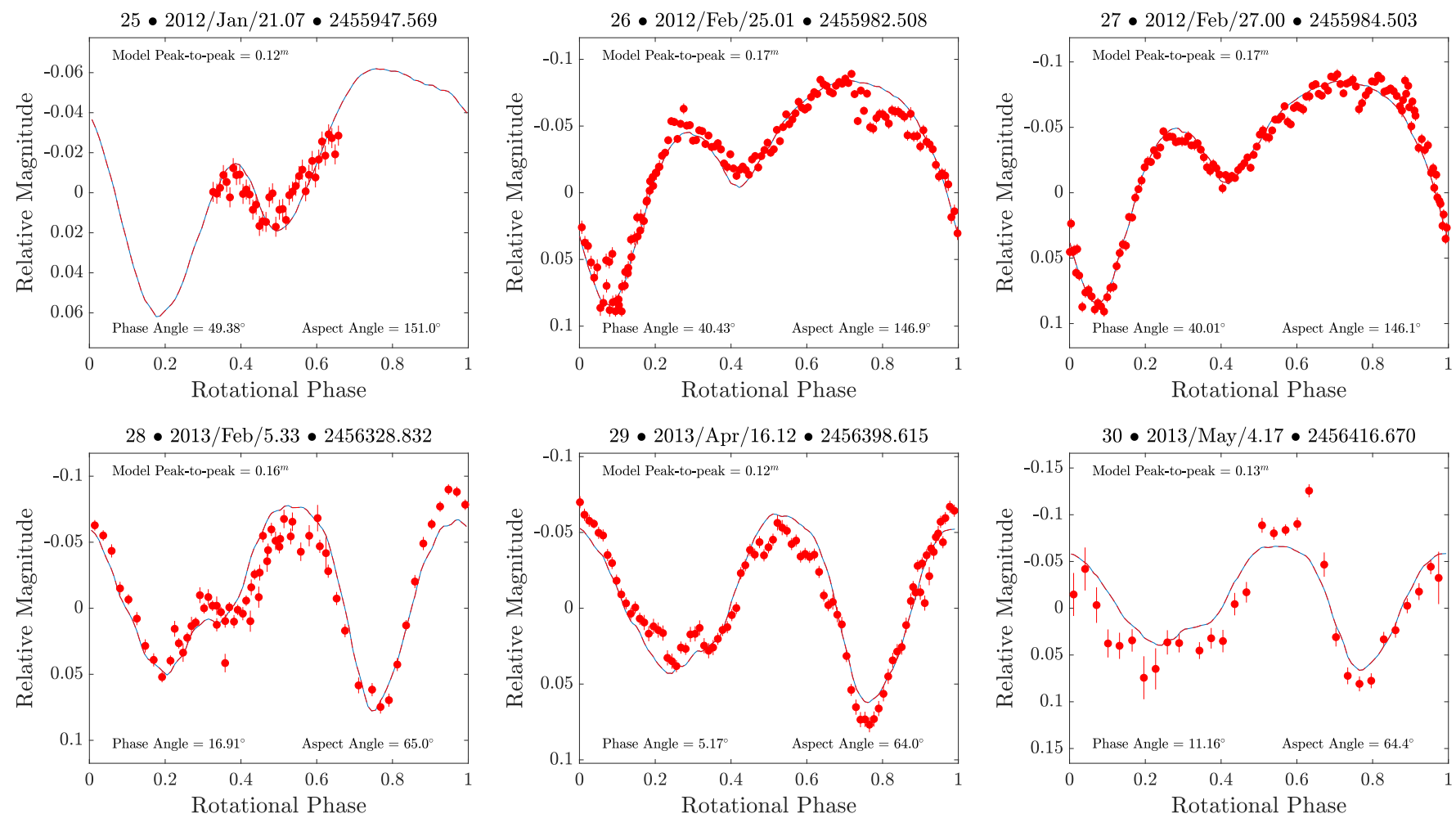

Fig. A.1. continued. 